\title{
Hierarchical Distributed Compressive Sensing for Simultaneous Sparse Approximation and Common Component Extraction
}

\author{
Arash Golibagh Mahyari, Selin Aviyente
}

\begin{abstract}
Simultaneous sparse approximation is a generalization of the standard sparse approximation, for simultaneously representing a set of signals using a common sparsity model. Distributed compressive sensing (DCS) framework has utilized simultaneous sparse approximation for generalizing compressive sensing to multiple signals. DCS finds the sparse representation of multiple correlated signals from compressive measurements using the common + innovation signal model. However, DCS is limited for joint recovery of a large number of signals since it requires large memory and computational time. In this paper, we propose a new hierarchical algorithm to implement the joint sparse recovery part of DCS more efficiently. The proposed approach is based on partitioning the input set and hierarchically solving for the sparse common component across these partitions. The numerical evaluation of the proposed method shows the decrease in computational time over DCS with an increase in reconstruction error. The proposed algorithm is evaluated for two different applications. In the first application, the proposed method is applied to video background extraction problem, where the background corresponds to the common sparse activity across frames. In the second application, a common network structure is extracted from dynamic functional brain connectivity networks.
\end{abstract}

Keywords

Compressive Sensing, Distributed Compressive Sensing, Electroencephalography, Functional Connectivity Network, Video Background Extraction, EEG, Motion Detection.

\section{INTRODUCTION}

Sparse signal approximation refers to the representation of a signal as a linear combination of a small subset of elements of a dictionary [1]. Sparse signal approximation usually considers one signal at a time, not taking into account the correlation within a group of signals. Simultaneous sparse approximation, a.k.a. joint sparse recovery, on the other hand, finds sparse representations of multiple signals collected through sensors monitoring the same environment simultaneously using a common dictionary [2], [3], [4]. Joint sparse recovery has been used in many

This work was in part supported by the National Science Foundation under Grant No. CCF-1422262.

Arash Golibagh Mahyari is with the Department of Electrical and Computer Engineering, Michigan State University, East Lansing, MI, 48824 USA (e-mail: mahyari@msu.edu).

Selin Aviyente is with the Department of Electrical and Computer Engineering, Michigan State University, East Lansing, MI, 48824 USA.

(C) 2016. This manuscript version is made available under the Elsevier user license http://www.elsevier.com/open-access/userlicense/1.0/ 
applications such as sensor networks [5], neuroelectromagnetic imaging [6], [7], source localization [8], and image restoration [9].

Different approaches to finding the common sparse representation among a set of signals have been proposed. Tropp et al. [2] proposed a greedy algorithm, i.e. simultaneous orthogonal matching pursuit (S-OMP), which extends orthogonal matching pursuit to joint sparse recovery. In [3], a convex relaxation approach was used to find the joint sparse approximation of multiple signals. Blanchard et al. [10] extended well-known sparse approximation methods, iterative hard thresholding, normalized iterative hard thresholding, hard thresholding pursuit, normalized hard thresholding pursuit, and Compressive Sampling Matching Pursuit, to the joint sparse recovery problem.

A closely related line of work was proposed in the compressive sensing community. Distributed compressive sensing (DCS) is an extension of compressive sensing to multiple observations problem [11], [12]. It simultaneously finds the sparse representation of a set of compressively sensed signals by assuming a common + innovation component model for signals. The common + innovation component model makes DCS a suitable tool to extract common component of highly correlated signals in many applications such as video processing [13], [14], [15] and time-varying networks [16]. However, a major problem with using DCS to extract the common component of a set of signals is that the size of the dictionary increases dramatically with the number of signals. For a set of $J N$-dimensional signals, the size of the required dictionary in DCS method to find the common component is $J N \times(J+1) N$, which demands huge memory and computational resources. In the joint sparse recovery literature, several methods for addressing this high computational complexity have been introduced. Lee et al [17] proposed orthogonal subspace matching pursuit (OSMP) for a new joint sparse recovery method, SA-MUSIC. OSMP is used in the the partial support recovery step of SA-MUSIC to provide a computationally efficient solution to joint sparse recovery with a performance guarantee. In [18], the performance of the joint sparse recovery of infinite measurement vectors (IMV), an infinite set of jointly sparse vectors, is improved. Instead of discretizing the entire infinite set of sparse vectors in IMV models, a reduced finite-dimensional problem is derived from which the common nonzero location set can be inferred exactly. In [19], $l_{1,2}$-norm penalty is used to enforce joint sparsity on the signals. The accelerated proximal gradient finds efficiently the joint sparse representation of the set of signals. Hyder and Mahata [20] proposed joint $l_{2,0}$ approximation algorithm (JLZA), an extension of the zero-norm approximation algorithm, to decrease the computational complexity while ensuring the robustness to the measurement noise. Even though these algorithms have improved the computational complexity of simultaneous sparse recovery problem, they all assume that the set of signals is sparse with respect to the same dictionary and the locations of the nonzero entries of the vector coefficient are the same among all signals while their values are different. However, DCS focuses on a more general problem with the assumption that the collection of signals have a common sparse component with respect to a basis while the innovation components are sparse with respect to another dictionary with the locations of the nonzero entries of the coefficient vector being different. Thus, these methods are not directly applicable to DCS.

In this paper, we propose a hierarchical algorithm to implement the joint sparse recovery part of DCS more efficiently. The proposed algorithm, called hierarchical distributed compressive sensing (H-DCS), partitions the set of signals into a small number of subsets, and finds the common component of each subset separately. The common components of the subsets are then used as a new set of signals to find the common component across all signals. The error bound and the computational complexity of DCS and hierarchical DCS are derived and compared, 
showing how hierarchical DCS outperforms DCS in terms of computational complexity. However, the error bound of hierarchical DCS is slightly larger than DCS due to the aggregated approximation errors across the iterations. Although this paper is focused on the two-stage implementation of H-DCS, it can be easily extended to more stages.

The proposed algorithm is evaluated for two different applications. In the first application, we consider the video background extraction problem which is of great importance in many automatic video content analysis applications such as surveillance video coding [21], motion detection [22], object tracking [23], [24], etc. Video background extraction aims to separate the moving objects, a.k.a. foreground, from the static objects, a.k.a. background, in order to facilitate the tracking of moving objects. Even though the subsequent steps of the background extraction are of more interest, the accuracy of the overall system depends on the performance of background extraction. Since the background scene does not change noticeably over time, it has a sparse representation with respect to a frequency basis such as DCT. Thus, H-DCS with discrete cosine transform (DCT) basis is used to extract the background scene.

In the second application, a method based on H-DCS is proposed to track dynamic functional connectivity brain networks over time. Functional connectivity (FC) has been extensively used to understand cognitive brain processes [25], [26]. One way to study FC networks in detail is through neuronal time series recorded from electroencephalogram (EEG) [25]. These time series are transformed to connectivity networks through bivariate synchronization measures between brain regions [27], where nodes correspond to distinct brain regions and edges to functional connectivity between them [28], [29], [30]. Evidence points to the fact that FC networks continuously form and destruct over multiple short-time intervals due to task demands, learning and anesthesia [31], [32], [33]. It is shown that these time-varying FC networks consist of a small number of distinct FC states corresponding to quasi-stationary time intervals [34], [35]. Thus, one way to track the dynamics of time-varying FC networks is to detect the change points in time where the FC networks change. FC networks generally consist of a background activity, which is common across all time steps, and a foreground network which corresponds to the transient activations [36]. The background activity is similar to the default mode network (DMN) identified from resting state fMRI, and varies slowly across time such that it can be assumed to be sparse with respect to frequency domain bases. Thus, the background activity (background FC pattern) is separated from the foreground by applying H-DCS with DCT as the basis of the common component. Since the innovation components are unique to each FC network, the dissimilarity of the innovation components of the consecutive FC networks is employed to detect the change points in the network structure. Once change points are detected, the time intervals can be summarized to obtain their common FC pattern through another stage of H-DCS algorithm.

\section{Distributed Compressive SENSING}

Distributed compressive sensing assumes that signals acquired across multiple sensors are sparse in a collection of bases, i.e. the set of signals is jointly sparse. Due to the inter-signal correlation, jointly sparse signals are usually assumed to be composed of a common sparse component which is shared by all signals, and an innovation component which is unique to each signal [37]. The encoding part of distributed compressive sensing is not different from compressive sensing in that each signal is separately projected onto some random, incoherent bases. However, the decoding is based on simultaneous sparse recovery of all signals, which can be used for various purposes 
including the common component extraction. In this paper, we only focus on the sparse recovery part of distributed compressive sensing.

\section{A. Joint Sparsity Model}

Let's assume that the set of signals $\Lambda=\left\{\mathbf{x}_{j} \in \mathbb{R}^{N} ; \forall j \in\{1,2, \ldots, J\}\right\}$ are jointly sparse. It is assumed that there is an inter-signal correlation among the signals. The joint sparsity model (JSM) [38], [12], which includes a common component $\mathbf{z}_{c} \in \mathbb{R}^{N}$ and an innovation component $\mathbf{z}_{j} \in \mathbb{R}^{N}$, can be written as:

$$
\mathbf{x}_{j}=\mathbf{z}_{c}+\mathbf{z}_{j}, \quad j=1,2, \ldots, J
$$

The common component represents the inter-signal correlation among the signals while the innovation component is unique to each signal. The common and innovation components of the set of signals $\Lambda$ are sparse with respect to two different sets of bases, $\phi_{c}$ and $\phi_{j}$, respectively, as:

$$
\begin{gathered}
\mathbf{z}_{c}=\phi_{c} \theta_{c}, \\
\mathbf{z}_{j}=\phi_{j} \theta_{j}, \quad j \in\{1,2, \ldots, J\},
\end{gathered}
$$

where $\theta_{c}$ and $\theta_{j}$ are the coefficient vectors, and the bases $\phi_{c}$ and $\phi_{j}$ are orthogonal. Since the signal $\mathbf{x}_{j}$ is sparse, the coefficient vectors have a small number of nonzero entities, $\left\|\theta_{c}\right\|_{0}=K_{c} \ll N$ and $\left\|\theta_{j}\right\|_{0}=K_{j} \ll N$.

In order to recover the sparse representation of the set of signals $\Lambda$, all signals are stacked to form a single optimization problem. Eq. 3 shows the compact representation of all signals and their sparse representations in matrix format.

$$
\begin{gathered}
\mathbf{X}=\mathbf{\Phi} \boldsymbol{\Theta}, \\
\boldsymbol{\Theta}=\left[\begin{array}{c}
\theta_{c} \\
\theta_{1} \\
\theta_{2} \\
\vdots \\
\theta_{J}
\end{array}\right], \quad \mathbf{X}=\left[\begin{array}{c}
\mathbf{x}_{1} \\
\mathbf{x}_{2} \\
\vdots \\
\mathbf{x}_{J}
\end{array}\right], \quad \mathbf{\Phi}=\left[\begin{array}{ccccc}
\phi_{c} & \phi_{1} & \mathbf{0} & \cdots & \mathbf{0} \\
\phi_{c} & \mathbf{0} & \phi_{2} & \cdots & \mathbf{0} \\
\vdots & \vdots & \ddots & \ddots & \vdots \\
\phi_{c} & \mathbf{0} & \cdots & \mathbf{0} & \phi_{J}
\end{array}\right],
\end{gathered}
$$

where $\mathbf{X} \in \mathbb{R}^{J N \times 1}, \boldsymbol{\Theta} \in \mathbb{R}^{J N \times 1}$, and $\mathbf{\Phi} \in \mathbb{R}^{J N \times(J+1) N}$.

The goal is to find the coefficient vector $\Theta$ such that the error between the signal $\mathbf{X}$ and the sparse representation $\Phi \Theta$ is minimized:

$$
\hat{\boldsymbol{\Theta}}=\arg \min \|\boldsymbol{\Theta}\|_{0} \text { s.t. }\|\mathbf{X}-\mathbf{\Phi} \Theta\| \leq \epsilon
$$

where $\epsilon$ is the bound on the recovery error. The optimization problem in Eq. 5 is $l_{0}$-norm minimization problem which is NP-hard and non-convex. The $l_{0}$-norm minimization is usually relaxed to $l_{1}$-norm minimization problem. However, in order to make the optimization problem more general and flexible, $\lambda$-weighted $l_{1}$-norm minimization problem is proposed [38], [12], as: 


$$
\hat{\boldsymbol{\Theta}}=\arg \min \lambda_{c}\left\|\theta_{c}\right\|_{1}+\sum_{j=1}^{J} \lambda_{j}\left\|\theta_{j}\right\|_{1} \text { s.t. }\|\mathbf{X}-\mathbf{\Phi} \Theta\| \leq \epsilon,
$$

where $\lambda_{c}, \lambda_{j} \geq 0$ are the weights of common and innovation components. In [39], [12], it is shown numerically that this modification improves the performance of the signal recovery. The weights, $\lambda_{j} \mathrm{~s}$, for the innovation components are usually selected to be the same. Without loss of generality, $\lambda_{j}$ s are selected to be equal to one and the optimal value of $\lambda_{c}$ is determined numerically [39]. The estimated coefficients, $\hat{\boldsymbol{\Theta}}$, are used for the sparse representation of the set of signals, or for recovering the common component $\mathbf{z}_{c}$.

\section{Signal Recovery USing Hierarchical Distributed Compressive Sensing}

\section{A. Joint Sparsity Model for Hierarchical Distributed Compressive Sensing}

The joint sparsity model assumed for the set of signals, $\Lambda=\left\{\mathbf{x}_{j} \in \mathbb{R}^{N} ; \forall j \in\{1,2, \ldots, J\}\right\}$, is the common + innovation component, as in Eq. 1. In this paper, in order to make the computation faster, we partition the set of signals, $\Lambda$, into $\Gamma$ arbitrary, non-overlapping subsets, $\Lambda_{\gamma}$, such that:

$$
\begin{aligned}
& \Lambda_{\gamma} \subset \Lambda \gamma=1,2, \ldots, \Gamma \\
& \Lambda= \bigcup_{\gamma=1}^{\Gamma} \Lambda_{\gamma}, \\
& \Lambda_{i} \cap \Lambda_{j}=\emptyset \quad \text { for } \quad i \neq j .
\end{aligned}
$$

Once the sparse recovery procedure is explained, the way these subsets are selected will be clarified in Section III-C

All signals $\mathbf{x}_{j} \in \Lambda$ have a common component, $\mathbf{z}_{c}$, due to the inter-signal correlation. Consequently, all signals within each subset $\mathbf{x}_{j} \in \Lambda_{\gamma} \subset \Lambda$ also have the same common component, $\mathbf{z}_{c}$. In addition, the signals within each subset may have additional inter-signal correlation, which leads to the common component within the subset $\Lambda_{\gamma}$. Thus, this model includes a global common component $\mathbf{z}_{c} \in \mathbb{R}^{N}$, a within-subset common component $\mathbf{w}_{c, \gamma} \in \mathbb{R}^{N}$, and a within-subset innovation component $\mathbf{w}_{j} \in \mathbb{R}^{N}$, as:

$$
\mathbf{x}_{j}=\mathbf{z}_{c}+\mathbf{w}_{c, \gamma}+\mathbf{w}_{j} \text { for } \mathbf{x}_{j} \in \Lambda_{\gamma}
$$

$\mathbf{u}_{\gamma}=\mathbf{z}_{c}+\mathbf{w}_{c, \gamma}$ is defined as the common component of the signals within the subset $\mathbf{x}_{j} \in \Lambda_{\gamma}$ while $\mathbf{z}_{c}$ is the global common component. Similarly, $\mathbf{z}_{j}=\mathbf{w}_{c, \gamma}+\mathbf{w}_{j}$ is the global innovation component. Since we assume the same joint sparsity model as Eq. 1. the common and innovation components are sparse on two sets of bases, $\phi_{c}$ and $\phi_{j}$, respectively, as:

$$
\begin{gathered}
\mathbf{u}_{\gamma}=\phi_{c} \theta_{u_{\gamma}} ; \quad \gamma=1,2, \ldots, \Gamma \\
\mathbf{w}_{j}=\phi_{j} \theta_{w_{j}} ; \quad\left\{j \mid \mathbf{x}_{j} \in \Lambda_{\gamma}\right\}
\end{gathered}
$$

where $\theta_{c}$ and $\theta_{j}$ are the coefficient vectors, with $\left\|\theta_{c}\right\|_{0}=K_{u_{\gamma}}$ and $\left\|\theta_{w_{j}}\right\|_{0}=K_{w_{j}}$. Fig. 1 shows the flowchart of the hierarchical DCS. 


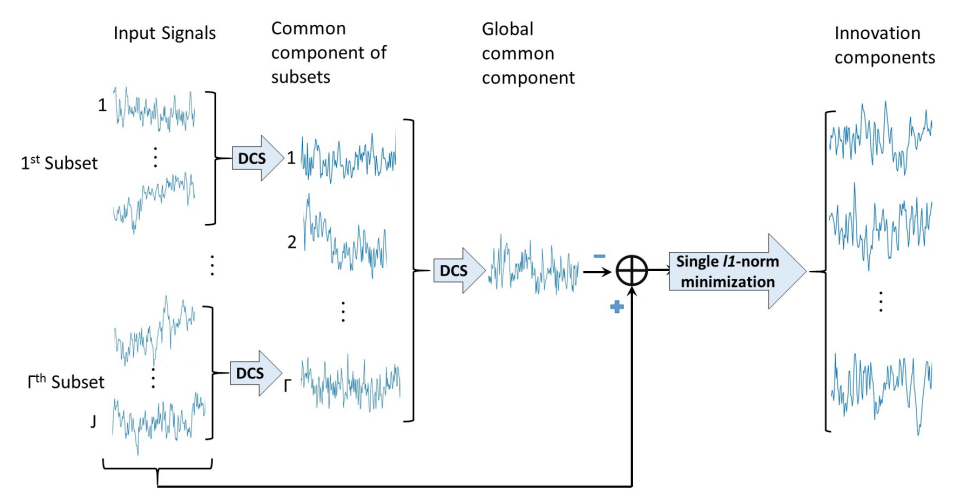

Fig. 1. Flowchart of the proposed method: Hierarchical DCS for two stages.

\section{B. Signal Recovery}

The goal is to extract the common component $\mathbf{z}_{c}$ when $J$ is large. First, the common component of each subset $\Lambda_{\gamma}$ is extracted similar to distributed compressive sensing through $l_{1}$-norm optimization algorithm by searching for the components with the sparsest coefficient vectors and minimum error. All signals of the subset $\Lambda_{\gamma}$ are stacked to form a vector as follows:

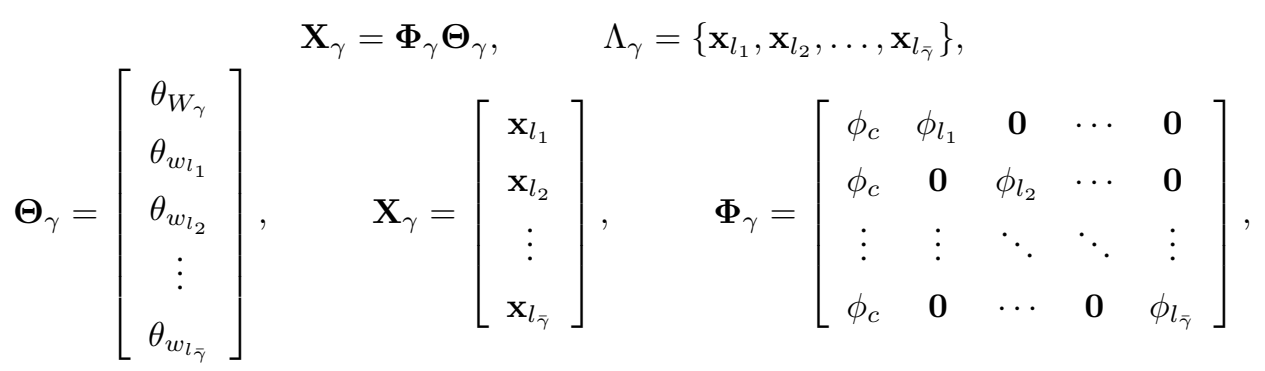

where $\left\{l_{1}, l_{2}, \ldots, l_{\bar{\gamma}}\right\}$ is the set of indices for the signals of the $\gamma$ th subset, and $\bar{\gamma}$ is the number of signals in the $\gamma$ th subset.

The common component for the $\gamma$ th subset, $\mathbf{u}_{\gamma}$, is estimated using $\hat{\mathbf{u}}_{\gamma}=\phi_{c} \hat{\theta}_{u_{\gamma}}$ through solving the following optimization equation:

$$
\hat{\boldsymbol{\Theta}}_{\gamma}=\arg \min \left\|\boldsymbol{\Theta}_{\gamma}\right\|_{1} \text { s.t. }\left\|\mathbf{X}_{\gamma}-\boldsymbol{\Phi}_{\gamma} \boldsymbol{\Theta}_{\gamma}\right\| \leq \epsilon .
$$

Once the common components of all subsets $\mathbf{u}_{\gamma} ; \forall \gamma \in\{1,2, \ldots, \Gamma\}$ are extracted, they are considered as a new set of jointly sparse signals with the common + innovation model: $\hat{\mathbf{u}}_{\gamma}=\mathbf{z}_{c}+\mathbf{w}_{c, \gamma}$, where $\mathbf{z}_{c}$ is their common component. $\mathbf{z}_{c}$ is recovered through another $l_{1}$-norm optimization algorithm.

After recovering the common component $\mathbf{z}_{c}$, the sparse representation of the innovation components, $\mathbf{z}_{j}$, are estimated through a single signal recovery problem:

$$
\hat{\theta}_{j}=\arg \min \left\|\theta_{j}\right\|_{1} \text { s.t. }\left\|\left(\mathbf{x}_{j}-\mathbf{z}_{c}\right)-\phi_{j} \theta_{j}\right\| \leq \epsilon .
$$


The pseudocode of the hierarchical DCS is presented in algorithm 1 .

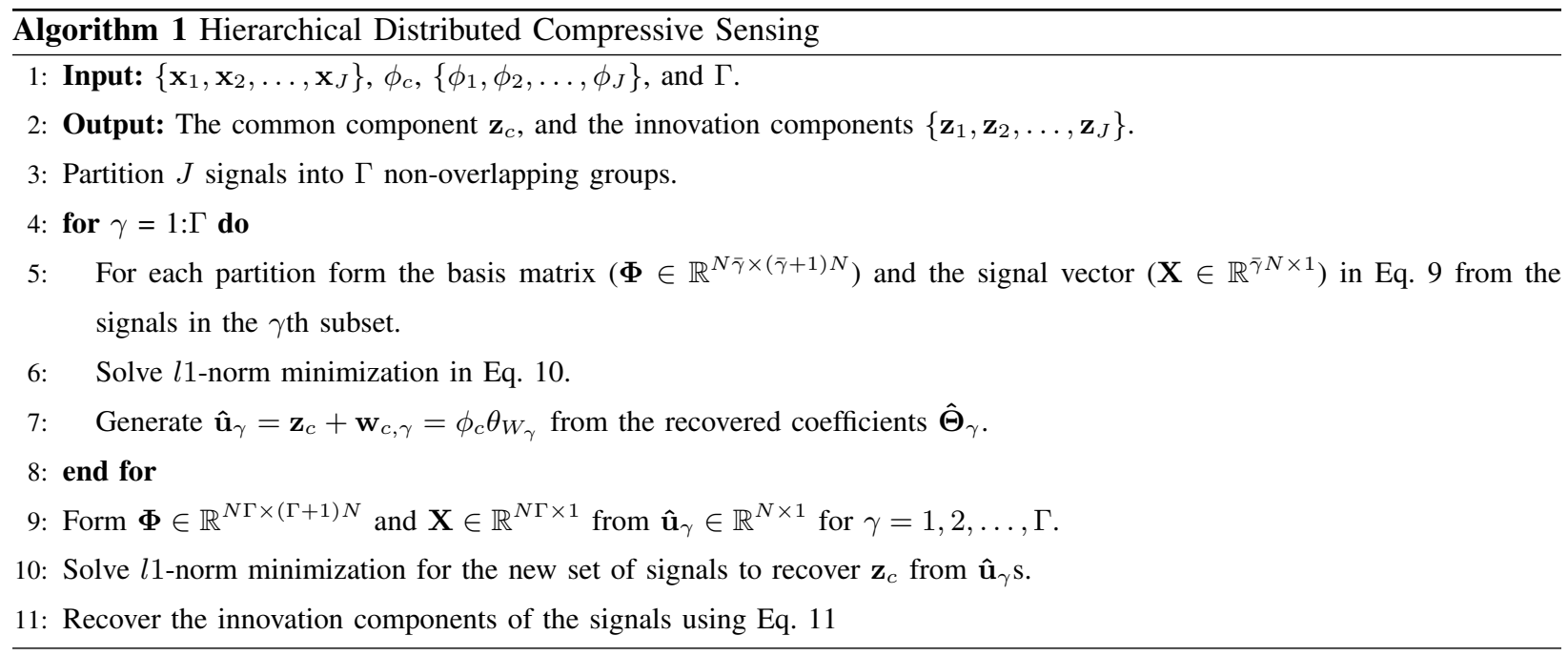

\section{Subset Selection}

In order to implement the proposed algorithm, the set of $J$ signals has to be split into $\Gamma$ groups. Here, we do not assume any a priori information regarding grouping the signals. Indeed, there is no need for the set of signals to be partitioned in a specific manner. Random grouping of the set of signals into $\Gamma$ subsets with equal size leads to the same results. We illustrate this through an example.

Assume we have a set of 4 jointly sparse signals, $\Lambda=\left\{\mathbf{x}_{1}, \mathbf{x}_{2}, \mathbf{x}_{3}, \mathbf{x}_{4}\right\}$. Our goal is to find the joint sparse representation of this set of signals. Assume there is an inter-signal correlation across the whole collection of signals, which is the global common component $\mathbf{z}_{c}$. In addition, assume that there is an additional within-subset common component $\mathbf{w}_{c, 1}$, between $\mathbf{x}_{1}$ and $\mathbf{x}_{2}$ which does not exist between these two signals and the remaining signals, $\mathbf{x}_{3}$ and $\mathbf{x}_{4}$. Note that this common component may also be equal to zero. Similarly, there is another withinsubset common component, $\mathbf{w}_{c, 2}$, between $\mathbf{x}_{3}$ and $\mathbf{x}_{4}$. However, in practice, we assume that we do not have $a$ priori information about the structure of the grouping and the inter-signal correlations. The set of signals can be written in terms of their components as follows:

$$
\begin{aligned}
& \mathbf{x}_{1}=\mathbf{z}_{c}+\mathbf{w}_{c, 1}+\mathbf{w}_{1}, \\
& \mathbf{x}_{2}=\mathbf{z}_{c}+\mathbf{w}_{c, 1}+\mathbf{w}_{2}, \\
& \mathbf{x}_{3}=\mathbf{z}_{c}+\mathbf{w}_{c, 2}+\mathbf{w}_{3}, \\
& \mathbf{x}_{4}=\mathbf{z}_{c}+\mathbf{w}_{c, 2}+\mathbf{w}_{4},
\end{aligned}
$$

where $\left\{\mathbf{w}_{1}, \mathbf{w}_{2}, \mathbf{w}_{3}, \mathbf{w}_{4}\right\}$ are the innovation components of the signals. Assuming that the common and innovation bases are orthogonal, Eq 12 can be rewritten as follows: 


$$
\begin{aligned}
& \mathbf{x}_{1}=\phi_{c} \theta_{c}+\phi_{j} \theta_{c, w_{1}}+\phi_{j} \theta_{w_{1}}, \\
& \mathbf{x}_{2}=\phi_{c} \theta_{c}+\phi_{j} \theta_{c, w_{1}}+\phi_{j} \theta_{w_{2}}, \\
& \mathbf{x}_{3}=\phi_{c} \theta_{c}+\phi_{j} \theta_{c, w_{2}}+\phi_{j} \theta_{w_{3}}, \\
& \mathbf{x}_{4}=\phi_{c} \theta_{c}+\phi_{j} \theta_{c, w_{2}}+\phi_{j} \theta_{w_{4}} .
\end{aligned}
$$

First, assume that we randomly partition these four signals into two subsets such that $\Lambda_{1}=\left\{\mathbf{x}_{1}, \mathbf{x}_{2}\right\}$ and $\Lambda_{2}=\left\{\mathbf{x}_{3}, \mathbf{x}_{4}\right\}$, the common components of the subsets will then be found through Algorithm 1 as

$$
\begin{aligned}
& \mathbf{u}_{1}=\mathbf{z}_{c}+\mathbf{w}_{c, 1}=\phi_{c} \underbrace{\left(\theta_{c}+\phi_{c}^{-1} \phi_{j} \theta_{c, w_{1}}\right)}_{\theta_{\mathbf{u}_{1}}}, \\
& \mathbf{u}_{2}=\mathbf{z}_{c}+\mathbf{w}_{c, 2}=\phi_{c} \underbrace{\left.\theta_{c}+\phi_{c}^{-1} \phi_{j} \theta_{c, w_{2}}\right)}_{\theta_{\mathbf{u}_{2}}} .
\end{aligned}
$$

By considering $\left\{\mathbf{u}_{1}, \mathbf{u}_{2}\right\}$ as a new set of jointly sparse signals, the common component of $\left\{\mathbf{u}_{1}, \mathbf{u}_{2}\right\}$ is extracted as $\mathbf{z}_{c}=\phi_{c} \theta_{c}$, which is the common component among all four signals.

Next, let's assume that the four signals are randomly categorized into two different subsets $\Lambda_{1}=\left\{\mathbf{x}_{1}, \mathbf{x}_{3}\right\}$ and $\Lambda_{2}=\left\{\mathbf{x}_{2}, \mathbf{x}_{4}\right\}$, then the common components of the subsets will be found as $\mathbf{u}_{1}=\mathbf{z}_{c}=\phi_{c} \theta_{c}$ and $\mathbf{u}_{2}=\mathbf{z}_{c}=\phi_{c} \theta_{c}$. If we construct a new subset of jointly sparse signals from $\mathbf{u}_{1}$ and $\mathbf{u}_{2}$, their common component is common among all four signals. Thus, no matter how the signals are categorized, the extracted common component is the true one, $\mathbf{z}_{c}$. If there is no inter-signal correlation within the subsets, i.e. $\mathbf{w}_{c, 1}=\mathbf{w}_{c, 2}=0$, the common component of the subsets (regardless of the partitioning) are $\mathbf{u}_{1}=\mathbf{z}_{c}$ and $\mathbf{u}_{2}=\mathbf{z}_{c}$.

\section{Computational Cost}

The proposed algorithm is significantly faster than existing simultaneous sparse representation algorithms. However, the computational cost mainly depends upon the algorithm used in solving the optimization problem. The optimization problems can be solved through either the vector-matrix multiplication or fast operators [40].

The joint sparse recovery of $J$ signals with $N$ samples requires a $J N \times(J+1) N$ dictionary, $\widetilde{\Phi}$, to be multiplied by a $(J+1) N$-dimensional coefficient vector $\Theta$. The cost of this vector-matrix multiplication is $\vartheta=\mathcal{O}((J+1) N \log ((J+1) N))$. The reason hierarchical DCS is able to reduce the computational cost significantly is that it splits the dictionary to $\Gamma$ matrices so that the optimization algorithm looks for the sparse representation of signals over a smaller set of bases.

In the worst case scenario, DCS implemented using the $l_{1}$-norm minimization costs $I \frac{(2(J+1) N)^{3}}{3}+\mathcal{O}\left(J(J+1) N^{2}\right)$, where $I$ is the number of iterations and assumed to be fixed for all cases [40]. The advantage of hierarchical DCS is that it can be implemented through multiple steps, and the $J$ signals can be partitioned into $\Gamma$ groups. In the following derivations, we will assume that one level of partitioning is adequate to compute the joint sparse recovery of signals, i.e. the common components of $\Gamma$ groups, $\mathbf{z}_{c}+\mathbf{z}_{\gamma}$, are calculated separately in the first stage and the global common component, $\mathbf{z}_{c}$, is extracted from the $\Gamma$ outcomes of the first stage. Each group contains $\frac{J}{\Gamma}$ signals in the first stage. Using the $l_{1}$-norm minimization, each group in the first stage requires $I \frac{\left(2\left(\frac{J}{\Gamma}+1\right) N\right)^{3}}{3}+\mathcal{O}\left(\frac{J}{\Gamma}\left(\frac{J}{\Gamma}+1\right) N^{2}\right)$ multiplications at most. These optimization problems are independent of each other and thus can be performed in 
parallel. In addition, the second stage cost is bounded by $I \frac{(2(\Gamma+1) N)^{3}}{3}+\mathcal{O}\left(\Gamma(\Gamma+1) N^{2}\right)$. Thus, the total computational cost required to recover the joint common component using Hierarchical DCS is upper bounded by

$$
I \frac{(2(J+\Gamma) N)^{3}}{3 \Gamma^{3}}+I \frac{(2(\Gamma+1) N)^{3}}{3}+\mathcal{O}\left(N^{2} \frac{J^{2}+\Gamma\left(\Gamma^{3}+\Gamma^{2}+J\right)}{\Gamma^{2}}\right) .
$$

For instance, let's suppose that we have $J=20$ jointly sparse signals with $N=256$. DCS with $l_{1}$-norm minimization costs $I 4.14 \times 10^{11}+\mathcal{O}\left(2.75 \times 10^{7}\right)$. If we divide the set of signals into $\Gamma=4$ groups, this cost becomes $I 4.43 \times 10^{10}+\mathcal{O}\left(3.28 \times 10^{6}\right)$. The computational cost of regular implementation is $9.36+\mathcal{O}(8.4)$ times greater than the hierarchical-based implementation, which is more than 10 times slower.

Using OMP for the $l 1$-norm optimization will result in a computational cost of $\frac{4(J N)^{3}}{3}+N^{3} J^{2}(J+1)+$ $\mathcal{O}(N(J+1))$ for DCS [40]. The hierarchical DCS based on OMP costs $\frac{4\left(\frac{J}{\Gamma} N\right)^{3}}{3}+N^{3} \frac{J^{2}}{\Gamma^{2}}\left(\frac{J}{\Gamma}+1\right)+\mathcal{O}\left(N\left(\frac{J}{\Gamma}+1\right)\right)$ in the first stage and $\frac{4(\Gamma N)^{3}}{3}+N^{3} \Gamma^{2}(\Gamma+1)+\mathcal{O}(N(\Gamma+1))$ in the second stage. Thus, the total cost is $\frac{4 J^{3}+\Gamma^{5} N^{3}}{3 \Gamma^{2}}+$ $\frac{N^{3}}{\Gamma^{2}}\left(J^{3}+J^{2} \Gamma+\Gamma^{5}+\Gamma^{4}\right)+\mathcal{O}(N(J+2 \Gamma+1))$. Table $\mathrm{I}$ shows the computational time for DCS and H-DCS for different values of $N$ and $J$. When $J=8$ and there is only one subset $\Gamma=1$, and the computational time of DCS and H-DCS are very close. However, as $J$ increases, the difference between computational time becomes more apparent.

TABLE I. THE COMPUTATIONAL COST OF DCS AND H-DCS USING OMP FOR DIFFERENT VALUES OF $J$ AND $N$. THE NUMBERS IN THE NUMERATOR SHOW THE COMPUTATIONAL COST OF DCS AND THE NUMBERS IN THE DENOMINATOR SHOW THAT OF H-DCS.

\begin{tabular}{|c|c|c|c|c|c|}
\hline$\frac{\check{I}}{\mathrm{I}}$ & $8 / 1$ & $\frac{16}{2}$ & $\frac{32}{4}$ & $\frac{64}{8}$ & $\frac{128}{16}$ \\
\hline 128 & $\frac{2.64 e^{9}+\mathcal{O}\left(1.15 e^{3}\right)}{1.21 e^{9}+\mathcal{O}\left(1.41 e^{3}\right)}$ & $\frac{2.06 e^{10}+\mathcal{O}\left(2.18 e^{3}\right)}{3.66 e^{8}+\mathcal{O}\left(1.66 e^{3}\right)}$ & $\frac{1.62 e^{11}+\mathcal{O}\left(4.22 e^{3}\right)}{3.13 e^{8}+\mathcal{O}\left(2.18 e^{3}\right)}$ & $\frac{1.29 e^{12}+\mathcal{O}\left(8.32 e^{3}\right)}{1.60 e^{9}+\mathcal{O}\left(3.20 e^{3}\right)}$ & $\frac{1.03 e^{13}+\mathcal{O}\left(1.65 e^{4}\right)}{1.20 e^{10}+\mathcal{O}\left(5.25 e^{3}\right)}$ \\
\hline 256 & $\frac{2.11 e^{10}+\mathcal{O}\left(2.30 e^{3}\right)}{9.70 e^{9}+\mathcal{O}\left(2.82 e^{3}\right)}$ & $\frac{1.65 e^{11}+\mathcal{O}\left(4.35 e^{3}\right)}{2.93 e^{9}+\mathcal{O}\left(3.33 e^{3}\right)}$ & $\frac{1.30 e^{12}+\mathcal{O}\left(8.45 e^{3}\right)}{2.51 e^{9}+\mathcal{O}\left(4.35 e^{3}\right)}$ & $\frac{1.03 e^{13}+\mathcal{O}\left(1.67 e^{4}\right)}{1.28 e^{10}+\mathcal{O}\left(6.40 e^{3}\right)}$ & $\frac{8.24 e^{13}+\mathcal{O}\left(3.30 e^{4}\right)}{9.60 e^{10}+\mathcal{O}\left(1.05 e^{4}\right)}$ \\
\hline 512 & $\frac{1.69 e^{11}+\mathcal{O}\left(4.61 e^{3}\right)}{7.76 e^{10}+\mathcal{O}\left(5.63 e^{3}\right)}$ & $\frac{1.32 e^{12}+\mathcal{O}\left(8.70 e^{3}\right)}{2.34 e^{10}+\mathcal{O}\left(6.66 e^{3}\right)}$ & $\frac{1.04 e^{13}+\mathcal{O}\left(1.69 e^{4}\right)}{2.00 e^{10}+\mathcal{O}\left(8.70 e^{3}\right)}$ & $\frac{8.27 e^{13}+\mathcal{O}\left(3.33 e^{4}\right)}{1.02 e^{11}+\mathcal{O}\left(1.28 e^{4}\right)}$ & $\frac{6.59 e^{14}+\mathcal{O}\left(6.60 e^{4}\right)}{7.68 e^{11}+\mathcal{O}\left(2.10 e^{4}\right)}$ \\
\hline 1024 & $\frac{1.35 e^{12}+\mathcal{O}\left(9.22 e^{3}\right)}{6.21 e^{11}+\mathcal{O}\left(1.13 e^{4}\right)}$ & $\frac{1.05 e^{13}+\mathcal{O}\left(1.74 e^{4}\right)}{1.88 e^{11}+\mathcal{O}\left(1.33 e^{3}\right)}$ & $\frac{8.32 e^{13}+\mathcal{O}\left(3.38 e^{4}\right)}{1.60 e^{11}+\mathcal{O}\left(1.74 e^{4}\right)}$ & $\frac{6.61 e^{14}+\mathcal{O}\left(6.66 e^{4}\right)}{8.19 e^{11}+\mathcal{O}\left(2.56 e^{4}\right)}$ & $\frac{5.27 e^{15}+\mathcal{O}\left(1.32 e^{5}\right)}{6.15 e^{12}+\mathcal{O}\left(4.20 e^{4}\right)}$ \\
\hline
\end{tabular}

\section{E. Error Bound}

Joint recovery of the sparse representation of a set of signals faster comes with the tradeoff of increased error. Since the problem of finding the sparse representation of the set of signals jointly is divided into $\Gamma$ different joint sparse recovery problems, this increases the reconstruction error.

Assuming we want to recover $\Theta$ from the observation vector $\mathbf{X}$, it is shown [41] that the reconstruction error from the $l_{1}$-norm optimization problem

$$
\min \|\boldsymbol{\Theta}\|_{1} \quad \text { s.t. } \quad\|\mathbf{X}-\Phi \Theta\| \leq \epsilon
$$

is bounded by:

$$
\|\hat{\mathbf{\Theta}}-\boldsymbol{\Theta}\|_{2} \leq C_{v} \epsilon
$$

where the constant $C_{v}$ depends on $\delta_{4 v}$ the $4 v$-restricted isometry constant of $\Phi$. 
However, most signals are not exactly sparse, rather approximately sparse. The coefficient vector $\Theta$ of such signals can be truncated with the $v$ largest coefficients, $\boldsymbol{\Theta}_{v}$. Then, the error bound for the approximately sparse signals follows:

$$
\|\hat{\boldsymbol{\Theta}}-\boldsymbol{\Theta}\|_{2} \leq C_{1, v} \epsilon+C_{2, v} \frac{\left\|\boldsymbol{\Theta}-\boldsymbol{\Theta}_{v}\right\|_{1}}{\sqrt{v}} .
$$

DCS recovers the common and innovation components jointly within one $l_{1}$-norm optimization problem, which means the vector $\Theta$ includes the sparse coefficients of all signals. Thus, the error bound for DCS joint recovery can be computed in terms of its components, $\mathbf{z}_{c}, \mathbf{w}_{c, \gamma}$ and $\mathbf{w}_{j}$, using Eq. 16, as:

$$
\left\|\hat{\boldsymbol{\Theta}}-\boldsymbol{\Theta}_{v}\right\|_{1}=\sum_{k=1}^{N}\left|z_{c}[k]-z_{c, v}[k]\right|+\sum_{\gamma=1}^{\Gamma} \sum_{k=1}^{N}\left|w_{c, \gamma}[k]-w_{c, \gamma, v}[k]\right|+\sum_{j=1}^{J} \sum_{k=1}^{N}\left|w_{j}[k]-w_{j, v}[k]\right|
$$

where we define the truncated vector $\boldsymbol{\Theta}_{v}$ as a vector with $J v$ nonzero entities. Each coefficient vector in $\hat{\boldsymbol{\Theta}}$ corresponding to $J$ signals are truncated using the $v$ largest values. The truncated vector $\boldsymbol{\Theta}_{v}$ is built from these $J$ truncated coefficient vectors. The upper bound for DCS is:

$$
\|\hat{\boldsymbol{\Theta}}-\boldsymbol{\Theta}\|_{2} \leq C_{1, v} \epsilon+\frac{C_{2, v}}{\sqrt{v}}\left(\sum_{k=1}^{N}\left|z_{c}[k]-z_{c, v}[k]\right|+\sum_{\gamma=1}^{\Gamma} \sum_{k=1}^{N}\left|w_{c, \gamma}[k]-w_{c, \gamma, v}[k]\right|+\sum_{j=1}^{J} \sum_{k=1}^{N}\left|w_{j}[k]-w_{j, v}[k]\right|\right)
$$

In order to calculate the upper error bound of the hierarchical DCS, we partition the dataset into $\Gamma$ subsets. Each subset requires a $l_{1}$-norm optimization problem, and the global common component is recovered through another $l_{1}$-norm optimization problem, which leads to a total of $\Gamma+1$ optimization problems.

Theorem 1: Assume that $\mathbf{X}=\Phi \Theta$ is a vector of $J$ jointly sparse signals following the signal model in Eq. 7 . where $\Phi$ is the dictionary built from $\phi_{c}, \psi_{i} \mathrm{~s}$, and $\phi_{j} \mathrm{~s}$. $\Phi$ has $v$-restricted isometry constant $\delta_{v}$, and $\|\mathbf{X}-\Phi \Theta\|_{2} \leq \epsilon$. Then, the error made by the hierarchical DCS recovering the jointly sparse representation of $J$ signals is bounded by:

$$
\|\hat{\boldsymbol{\Theta}}-\boldsymbol{\Theta}\|_{2} \leq C_{1, v} \epsilon+\frac{C_{2, v}}{\sqrt{v}}\left(2 \sum_{k=1}^{N}\left|z_{c}[k]-z_{c, v}[k]\right|+2 \sum_{\gamma=1}^{\Gamma} \sum_{k=1}^{N}\left|w_{c, \gamma}[k]-w_{c, \gamma, v}[k]\right|+\sum_{j=1}^{J} \sum_{k=1}^{N}\left|w_{j}[k]-w_{j, v}[k]\right|\right)
$$

where $C_{1, v}$ and $C_{2, v}$ depend on $\delta_{4 v}[41]$.

Proof:

Following the upper error bound provided by Eq. 16 , the upper error bound for the $\gamma$ th subset is:

$$
\begin{gathered}
\left\|\hat{\boldsymbol{\Theta}}_{\gamma}-\boldsymbol{\Theta}_{\gamma}\right\|_{2} \leq C_{1, v, \gamma} \epsilon+\frac{C_{2, v, \gamma}}{\sqrt{v}}\left(\sum_{k=1}^{N}\left|z_{c}[k]-z_{c, v}[k]\right|\right. \\
\left.+\sum_{k=1}^{N}\left|w_{c, \gamma}[k]-w_{c, \gamma, v}[k]\right|+\sum_{j \in \gamma \text { th subset }} \sum_{k=1}^{N}\left|w_{j}[k]-w_{j, v}[k]\right|\right) .
\end{gathered}
$$

The constants $C_{1, v, \gamma}$ and $C_{2, v, \gamma}$ depend on the $v$-restricted isometry constant, $\delta_{v, \gamma}$, of the matrix $\Phi_{\gamma}$. Since $\Phi_{\gamma}$ is a submatrix of $\Phi$, the relationship between the local isometry $\delta_{v, \gamma}$ and the global isometry $\delta_{v}$, the $v$-restricted isometry constant of the matrix $\Phi$, is as follows [42]: 


$$
\delta_{v}=\sup _{\gamma} \delta_{v, \gamma}
$$

Therefore, we can conclude that:

$$
\begin{gathered}
\left\|\hat{\boldsymbol{\Theta}}_{\gamma}-\boldsymbol{\Theta}_{\gamma}\right\|_{2} \leq C_{1, v} \epsilon+\frac{C_{2, v}}{\sqrt{v}}\left(\sum_{k=1}^{N}\left|z_{c}[k]-z_{c, v}[k]\right|+\sum_{k=1}^{N}\left|w_{c, \gamma}[k]-w_{c, \gamma, v}[k]\right|\right. \\
\left.+\sum_{j \in \gamma \text { th subset }} \sum_{k=1}^{N}\left|w_{j}[k]-w_{j, v}[k]\right|\right) \forall \gamma \in\{1,2, \ldots, \Gamma\} .
\end{gathered}
$$

After calculating the sparse representation of $\Gamma$ subsets, the last $l_{1}$-norm optimization step finds the common part among their outputs. The recovery error from this last step is calculated similarly using Eq. 16, as follows:

$$
\left\|\hat{\boldsymbol{\Theta}}_{c}-\boldsymbol{\Theta}_{c}\right\|_{2} \leq C_{1, v} \epsilon+\frac{C_{2, v}}{\sqrt{v}}\left(\sum_{k=1}^{N}\left|z_{c}[k]-z_{c, v}[k]\right|+\sum_{\gamma=1}^{\Gamma} \sum_{k=1}^{N}\left|w_{c, \gamma}[k]-w_{c, \gamma, v}[k]\right|\right) .
$$

Therefore, the total recovery error for the proposed algorithm is bounded by:

$$
\|\hat{\boldsymbol{\Theta}}-\boldsymbol{\Theta}\|_{2} \leq C_{1, v} \epsilon+\frac{C_{2, v}}{\sqrt{v}}\left(2 \sum_{k=1}^{N}\left|z_{c}[k]-z_{c, v}[k]\right|+2 \sum_{\gamma=1}^{\Gamma} \sum_{k=1}^{N}\left|w_{c, \gamma}[k]-w_{c, \gamma, v}[k]\right|+\sum_{j=1}^{J} \sum_{k=1}^{N}\left|w_{j}[k]-w_{j, v}[k]\right|\right)
$$

Comparing the error bounds of DCS and hierarchical DCS in Eq. 18 and Eq. 19 imply that the upper error bound of hierarchical DCS is larger than that of DCS by $\frac{C_{2, v}}{\sqrt{v}}\left(\sum_{k=1}^{N}\left|z_{c}[k]-z_{c, v}[k]\right|+\sum_{\gamma=1}^{\Gamma} \sum_{k=1}^{N}\left|w_{c, \gamma}[k]-w_{c, \gamma, v}[k]\right|\right)$.

More recently, Needell et. al [43] proposed Regularized Orthogonal Matching Pursuit (ROMP) to find a sparse representation of signals with a tighter error bound. The error bound of DCS when ROMP algorithm is used for sparse recovery is equal to:

$$
\begin{gathered}
\|\hat{\boldsymbol{\Theta}}-\boldsymbol{\Theta}\|_{2} \leq C \sqrt{\log v}\left(\|\epsilon\|_{2}+\frac{1}{\sqrt{v}}\left(\sum_{k=1}^{N}\left|z_{c}[k]-z_{c, v}[k]\right|\right.\right. \\
\left.\left.+\sum_{\gamma=1}^{\Gamma} \sum_{k=1}^{N}\left|w_{c, \gamma}[k]-w_{c, \gamma, v}[k]\right|+\sum_{j=1}^{J} \sum_{k=1}^{N}\left|w_{j}[k]-w_{j, v}[k]\right|\right)\right),
\end{gathered}
$$

where $C$ is a constant, and $\epsilon$ is the noise, which is zero in the noiseless situation.

Theorem 2: Assume that $\mathbf{X}=\Phi \Theta$ is a vector of $J$ jointly sparse signals following the signal model in Eq. 7 . where $\Phi$ is the dictionary built from $\phi_{c}, \psi_{i} \mathrm{~s}$, and $\phi_{j} \mathrm{~s}$. Then, the error made by the hierarchical DCS recovering the jointly sparse representation of $J$ signals using ROMP algorithm is bounded by:

$$
\begin{gathered}
\|\hat{\boldsymbol{\Theta}}-\boldsymbol{\Theta}\|_{2} \leq C \sqrt{\log v}\left(\|\epsilon\|_{2}+\frac{1}{\sqrt{v}}\left(\sum_{k=1}^{N}\left|z_{c}[k]-z_{c, v}[k]\right|\right.\right. \\
\left.\left.+\sum_{k=1}^{N}\left|w_{c, \gamma}[k]-w_{c, \gamma, v}[k]\right|+\frac{1}{2} \sum_{j=1}^{J} \sum_{k=1}^{N}\left|w_{j}[k]-w_{j, v}[k]\right|\right)\right),
\end{gathered}
$$

where $C$ is a constant, and $\epsilon$ is the noise, which is zero in the noiseless situation [43].

Proof:

The upper error bound for the $\gamma$ th subset is:

$$
\begin{gathered}
\left\|\hat{\boldsymbol{\Theta}}_{\gamma}-\boldsymbol{\Theta}_{\gamma}\right\|_{2} \leq C \sqrt{\log v}\left(\|\epsilon\|_{2}+\frac{1}{\sqrt{v}}\left(\sum_{k=1}^{N}\left|z_{c}[k]-z_{c, v}[k]\right|\right.\right. \\
\left.\left.+\sum_{k=1}^{N}\left|w_{c, \gamma}[k]-w_{c, \gamma, v}[k]\right|+\sum_{j \in \gamma \text { th subset }} \sum_{k=1}^{N}\left|w_{j}[k]-w_{j, v}[k]\right|\right)\right) .
\end{gathered}
$$

After calculating the sparse representation of $\Gamma$ subsets, the last $l_{1}$-norm optimization step finds the common part among their outputs. The recovery error from this last step is calculated similarly as follows: 


$$
\left\|\hat{\boldsymbol{\Theta}}_{c}-\boldsymbol{\Theta}_{c}\right\|_{2} \leq C \sqrt{\log v}\left(\|\epsilon\|_{2}+\frac{1}{\sqrt{v}}\left(\sum_{k=1}^{N}\left|z_{c}[k]-z_{c, v}[k]\right|+\sum_{\gamma=1}^{\Gamma} \sum_{k=1}^{N}\left|w_{c, \gamma}[k]-w_{c, \gamma, v}[k]\right|\right)\right) .
$$

The total recovery error of H-DCS calculated using ROMP is bounded by:

$$
\begin{gathered}
\|\hat{\boldsymbol{\Theta}}-\boldsymbol{\Theta}\|_{2} \leq C \sqrt{\log v}\left(\|\epsilon\|_{2}+\frac{1}{\sqrt{v}}\left(\sum_{k=1}^{N}\left|z_{c}[k]-z_{c, v}[k]\right|\right.\right. \\
\left.\left.+\sum_{k=1}^{N}\left|w_{c, \gamma}[k]-w_{c, \gamma, v}[k]\right|+\frac{1}{2} \sum_{j=1}^{J} \sum_{k=1}^{N}\left|w_{j}[k]-w_{j, v}[k]\right|\right)\right) .
\end{gathered}
$$

\section{F. Numerical Performance Evaluation}

In this section, we present the numerical evaluation of the proposed method to show the improvement of Hierarchical DCS with respect to DCS. A real-world scenario introduced in [12] is used here to compare the computational cost and the reconstruction error of Hierarchical DCS and DCS, numerically.

In order to generate a set of sparse signals with inter-signal correlation, the common and innovation components of the joint sparsity model (Eq. 1) are created separately. To create the sparse common component, the location of the nonzero entries of the coefficient vector, $\theta_{c}$, are selected randomly with the sparsity $K_{c} \sim \operatorname{Binomial}\left(N, p_{c}=\frac{1}{5}\right)$. The value of the selected nonzero coefficients are chosen from $N(\mu, \sigma)$. DCT bases is chosen as the common component bases, $\Phi_{c} \in \mathbb{R}^{N \times N}$. Then, the common component is obtained through $\mathbf{z}_{c}=\Phi_{c} \theta_{c}$. Similarly, the location of the nonzero entries of the coefficient vectors of the innovation components, $\theta_{j}, \forall j$, are chosen randomly with the sparsity $K_{j} \sim \operatorname{Binomial}\left(N, p_{j}=\frac{1}{5}\right)$. The value of nonzero coefficients are chosen from $N(\mu, \sigma)$. The $N \times N$ Haar basis is selected as the innovation component dictionary $\Phi_{j}$. The $j$ th signal in the collection is then constructed as $x_{j}=\Phi_{c} \theta_{c}+\Phi_{j} \theta_{j}$.

In the first experiment, the length of the signal, $N$, is kept constant and equal to 128 samples. In order to consider the effect of the number of signals, $J$, on the computational cost, the number of signals, $J$, is selected from $\{8,16,24,32,40,48,56,64,72,80\}$. DCS is used to find the common and innovation components of $J$ jointly distributed signals. In order to use the hierarchical DCS, the number of subsets, $\Gamma$, is selected regardless of the value of $J$ such that $\frac{J}{\Gamma}=8$. The experiment is repeated 1000 times. The computational cost and error are calculated for both methods. Fig. 2 shows the mean $+/-$ the standard deviation of the computational time required to calculate DCS and hierarchical DCS. Fig. 3 shows the mean +/- the standard deviation of mean squared error (MSE) of both DCS and hierarchical DCS. As it can be seen, the computational cost of DCS grow exponentially as the number of signals increases. Yet, the hierarchical DCS requires less amount of time with smaller standard deviation. On the other hand, MSE of hierarchical DCS is becoming saturated, and in this experience in the worst case the MSE differences between two methods is $9 \times 10^{-4}$. Thus, if there is no restriction on the time to calculate the joint sparsity model and any memory limitations, DCS works better since MSE is smaller. However, for larger values of $J$, Hierarchical DCS computes the common + innovation joint sparsity model much faster at the cost of slightly increased MSE.

In the second experiment, $J$ jointly sparse signals are generated in the same way as described in the first experiment. However, the common and innovation bases are chosen to be identical and equal to $N \times N$ DCT. The computational cost and MSE are calculated for both DCS and hierarchical DCS. Fig. 4 shows the mean $+/-$ the 


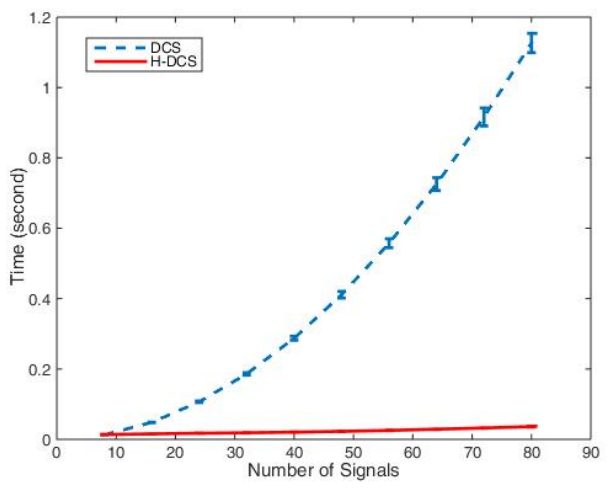

Fig. 2. The mean +/- the standard deviation of the computational time required to calculate DCS and hierarchical DCS when the bases of the common components is DCT and the bases of the innovation components is Haar.

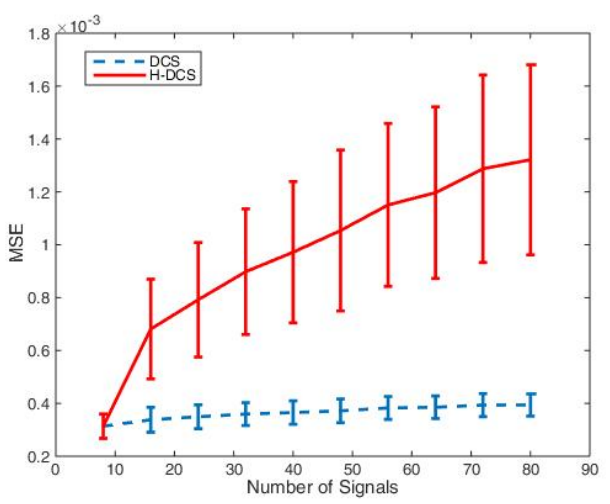

Fig. 3. The mean +/- the standard deviation of MSE of both DCS and hierarchical DCS when the bases of the common components is DCT and the bases of the innovation components is Haar.

standard deviation of the computational time required to calculate DCS and hierarchical DCS. Fig. 5 shows the mean +/- the standard deviation of MSE of both algorithms.

Apparent from Fig. 2 and 4 the computational time of DCS is larger on average in addition to its standard deviation in the second experiment compared to the first one. Similarly, the mean and standard deviation of MSE for DCS decrease for the second experiment while they stay the same for H-DCS. Therefore, in situations where the common and innovation components can be assumed to be sparse with respect to the same set of bases, the performance of H-DCS improves more compared to DCS in terms of the computational time and reconstruction error.

In the third example, we investigate the effect of the noise on H-DCS and compare the results with DCS. $J$ jointly sparse signals are generated in the same way as described in the first and second experiments. The common and innovation bases are chosen to be identical and equal to $N \times N$ DCT. However, the number of signals is fixed and equal to $J=16$ in this experiment so that the only variable is the signal-to-Noise ration (SNR). The experiment is performed for 10 different SNR values and repeated 1000 times for each SNR value. Fig. 6 and Fig. 7 show 


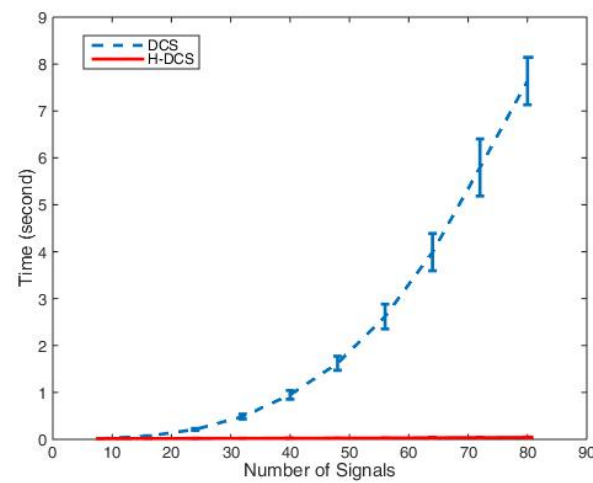

Fig. 4. The mean $+/$ - the standard deviation of the computational time required to calculate DCS and hierarchical DCS when the bases of the common components and the innovation components are identical (DCT).

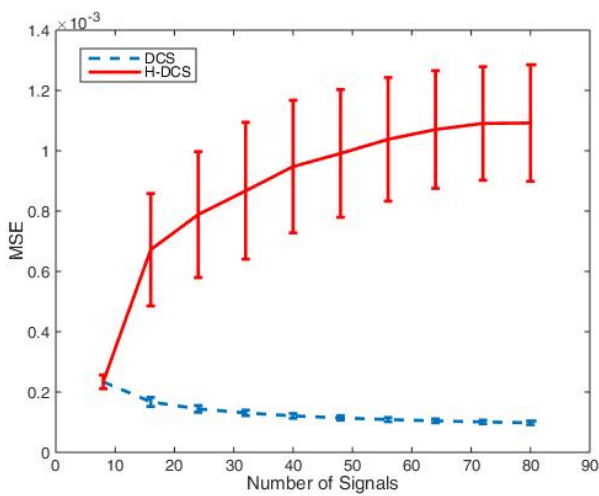

Fig. 5. The mean +/- the standard deviation of MSE of both DCS and hierarchical DCS when the bases of the common components and the innovation components are identical (DCT).

the computation time and MSE values of DCS and H-DCS for different SNR values. H-DCS outperforms DCS in terms of computational time for all SNR values. On the other hand, MSE of DCS is less than that of H-DCS for larger values of SNR whereas MSE of DCS becomes larger than that of H-DCS with the decrease in the SNR value. When SNR values are large, DCS is able to recover the sparse representation of the original signals while H-DCS introduces some noise into the recovered signals. However, as SNR values decreases, H-DCS outperforms DCS since H-DCS first focuses on the recovery of the sparse representation of the common component. In the first level of the implementation, H-DCS filters out the innovation components and a part of the noise. Then, in the second stage, H-DCS filters out the remaining noise and innovation components. Once the common component is recovered, in the final stage, H-DCS recovers the innovation components by filtering out the noise through the sparse recovery. Thus, H-DCS consists of three levels of sparse approximation, each acting as a noise filtering by finding the low-dimensional representation of the signals. 


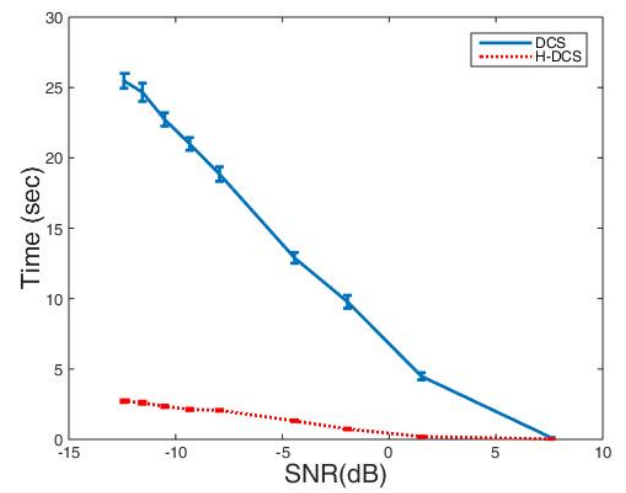

Fig. 6. The mean +/- the standard deviation of the computational time required to calculate DCS and hierarchical DCS when the bases of the common components and the innovation components are identical (DCT) and the signals are contaminated by noisy.

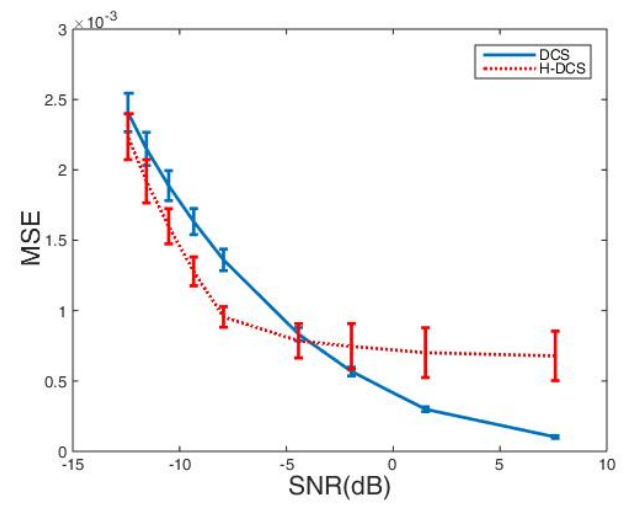

Fig. 7. The mean +/- the standard deviation of MSE for both DCS and hierarchical DCS when the bases of the common components and the innovation components are identical (DCT) for noisy signals with varying SNR values.

\section{APPLICATIONS}

\section{A. Video Background Extraction}

Recently, DCS has been used to extract the background scene in video as it can be thought of as the common component of all frames [13]. For example, Do et al. [14] designed a real-time video coding system based on distributed compressive sensing. In this section, we use the H-DCS to extract the background scene from a sequence of images in order to find moving objects. The proposed H-DCS overcomes restrictions such as time and memory, which helps to extract the background scene of a longer sequence of images. The experimental results are compared with one of the widely used video background extraction methods, called visual background extractor (ViBe) [44] which uses a random policy to estimate the sample-based background.

1) Implementation: In the first experiment, a sequence of $9,512 \times 512$-images of toy vehicles is chosen for background extraction purposes [45], where the images consist of a common background scene and moving objects. The images are downsampled to $80 \times 80$ due to computation time and memory limitations. All 9 images are 
vectorized, $\Lambda=\left\{\mathbf{x}_{j} \in \mathbb{R}^{6400} ; \forall j \in\{1,2, \ldots, 9\}\right\}$ with $\mathbf{x}_{j}=\mathbf{b}_{c}+\mathbf{f}_{j}$ where $\mathbf{b}_{c}$ is the background scene and $\mathbf{f}_{j}$ is the moving object corresponding to the $j$ th frame. The $6400 \times 6400$ identity matrix $\mathbf{I}_{6400}$ is used as the bases for the common and innovation components (background and foreground), $\phi_{c}=\phi_{j}=\mathbf{I}_{6400}$. The background of the set of images, $\Lambda$, is extracted first using DCS method.

The same set of $80 \times 80$ images is then used to evaluate the performance of the H-DCS algorithm. The set of 9 images are partitioned into $\Gamma=3$ sets by placing the consecutive images into the same subset. The identity matrix is selected as the bases for the common and innovation components, $\phi_{c}=\phi_{j}=\mathbf{I}_{6400}$. The common component of each subset is extracted separately through the $l 1$-norm optimization problem (Eq. 10, yielding $\mathbf{u}_{\gamma}, \gamma \in\{1,2,3\}$. We use the common components of these three subsets to extract the global common component, $\mathbf{b}_{c}$, solving another $l 1$-norm optimization problem.

H-DCS is also applied to the same sequence of images downsampled to $128 \times 128$. All images are vectorized to form the signal set $\Lambda=\left\{\mathbf{x}_{j} \in \mathbb{R}^{6400} ; \forall j \in\{1,2, \ldots, 9\}\right\}$. Every three consecutive images are grouped into one subset. Fig. 8 shows three frames from the original sequence and the foreground extracted by DCS and H-DCS for different image sizes. The original DCS is not capable of extracting the background from images with size $128 \times 128$ due to memory and computational time requirements. The downsampled background obtained from hierarchical DCS is compared with the background extracted using DCS. Table II shows the computational time and peak signal to noise ration (PSNR) for this set of experiments. In order to calculate PSNR, the background extracted with DCS is considered as the groundtruth and compared with that extracted from H-DCS. However, in the case of $128 \times 128$ set of images, DCS is not capable of extracting the background of the images due to memory and computational time restrictions. Thus, the downsampled background obtained from H-DCS is compared with the background extracted using DCS in order to calculate PSNR. By comparing the third and fourth rows of Fig. 8 . we can see that there is some misidentification of the moving objects in the hierarchical DCS method, which can be explained through the larger error bound of hierarchical DCS. In the fourth row, there are some artifacts or distortion between the two vehicles, mostly due to the different location of the vehicles. These distortions show the movement of the vehicles in the sequence of the images. These distortions do not exist in the foreground detected using DCS. However, as the dimension of the images grows, the distortion from H-DCS decreases (the fifth row of Fig. 8) since there are a larger number of coefficients in $\Theta$ which allows the optimization problem to find a better combination of coefficients to reduce the error. This explains the improvement in MSE and PSNR in Table III Note that the distortion can also be decreased through tightening the conditions of the optimization problem, which will slightly increase the convergence time.

TABLE II. THE COMPUTATIONAL COST AND PSNR FOR THE TOY-VEHICLE EXPERIMENT

\begin{tabular}{lccc}
\hline \hline \multirow{2}{*}{ Method } & \multicolumn{2}{c}{ Computational Cost $(\min )$} & \multirow{2}{*}{ PSNR $(\mathrm{dB})$} \\
\cline { 2 - 3 } & H-DCS & DCS & \\
\hline $80 \times 80$ frames & 28 & 141 & 33.33 \\
$128 \times 128$ frames & 76 & - & 36 \\
\hline
\end{tabular}




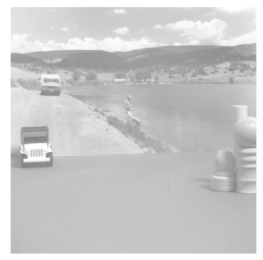

(a)

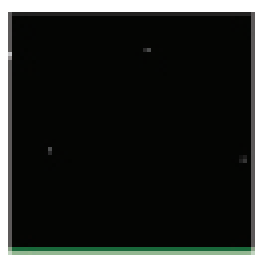

(g)

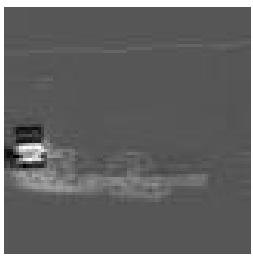

(m)

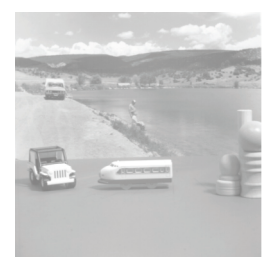

(b)

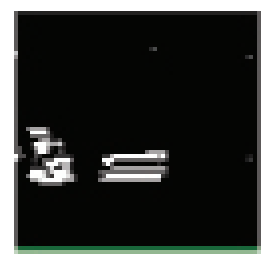

(h)

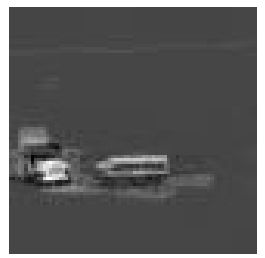

(n)

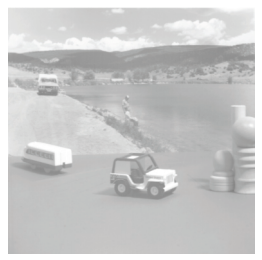

(c)

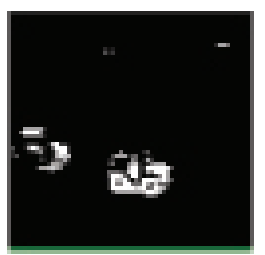

(i)

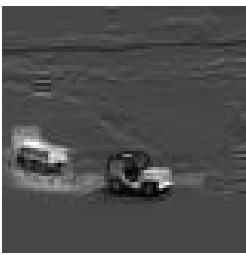

(o)

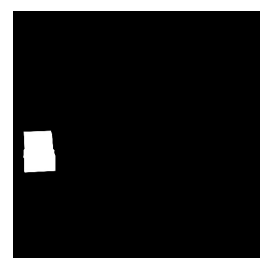

(d)

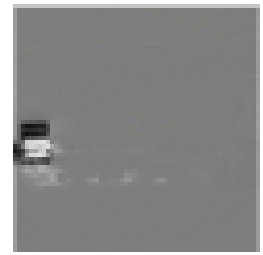

(j)

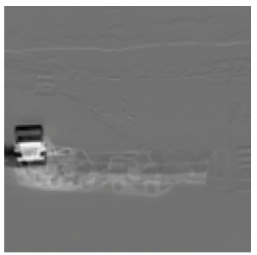

(p)

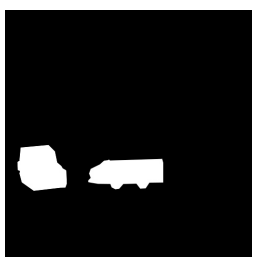

(e)

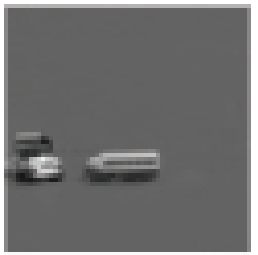

(k)

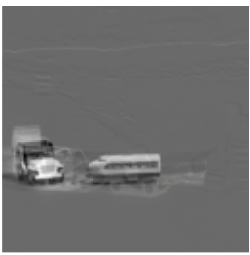

(q)

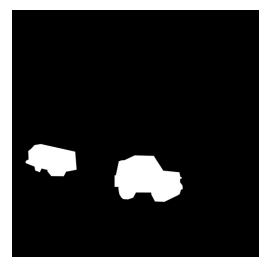

(f)

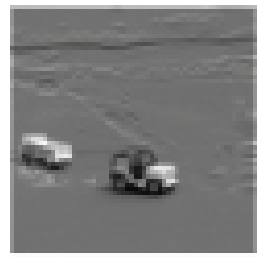

(1)

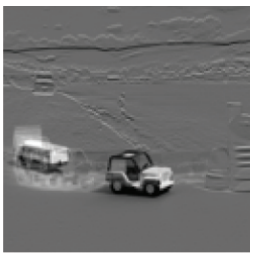

(r)

Fig. 8. The toy vehicle experiment: first column shows three original frames of the sequence, the second column is the ground truth for the moving objects, the third column is the corresponding results obtained by ViBe [44] with frames resized to $80 \times 80$, the fourth column is the detected moving objects using DCS with frames resized to $80 \times 80$, the fifth column is the detected moving objects using H-DCS with frames resized to $80 \times 80$, the sixth column is the detected moving objects using H-DCS with frames resized to $128 \times 128$.

\section{B. Functional Brain Connectivity Network State Representation}

In this section, we propose to use H-DCS to track dynamic FC networks constructed from multichannel EEG data collected during a study of Error-related negativity (ERN). The dynamic network is converted to signals or vectors at each time point and the H-DCS framework is applied to this collection of signals to first identify change points in connectivity and then extract the common sparse components for network summarization.

1) Data: EEG data from 62-channels was collected in accordance with the $10 / 20$ system on a Neuroscan Synamps 2 system (Neuroscan, Inc.) at a sampling rate of $128 \mathrm{~Hz}$. This study included 91 undergraduate students (34 male) from the University of Minnesota. Full methodological details of the recording are available in a previous paper [46]. The task was a common speeded-response letter (H/S) flanker, where error response-locked trials from each subject was utilized. The EEG data are pre-processed by the spherical spline current source density (CSD) waveforms to sharpen event-related potential (ERP) scalp topographies and reduce volume conduction [47].

The ERN is a brain potential response that occurs following performance errors in a speeded reaction time task usually 25-75 ms after the response [48]. Previous work [49], [50] indicates that there is increased phase synchrony associated with ERN for the theta frequency band $((4,7) \mathrm{Hz})$ and ERN time window $((25,75) \mathrm{ms})$ for Error responses compared to Correct responses [50]. Moreover, this increased synchrony is observed between the 


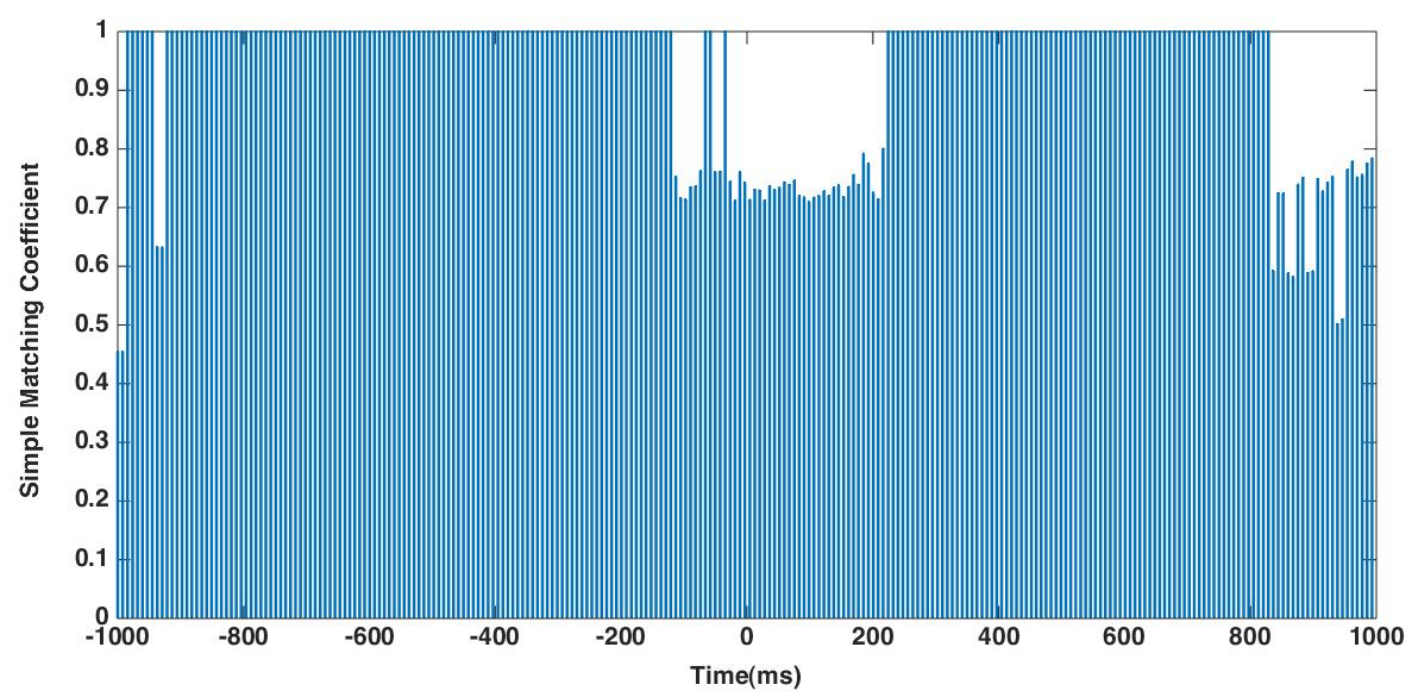

Fig. 9. Simple Matching Coefficient: similarity between the support of the coefficients of the consecutive $\hat{\mathbf{g}}(t)$.

electrodes corresponding to the lateral prefrontal cortex (IPFC) and medial prefrontal cortex (mPFC) [49].

2) Functional Connectivity Network: FC networks represent transient synchronization between distant and specific neural populations, leading to human cognition [51], [52], [26]. A common way to quantify functional connectivity is through phase synchronization measures. In this paper, we used a recently introduced RID-Rihaczek phase synchrony measure to quantify the pairwise connectivity across electrodes [53].

Once the pairwise synchrony phase locking values (PLV), $P L V_{(i, j)}(t, \omega)$, are computed at each time and frequency point, we can construct a time-varying connectivity graph $\{\mathbf{G}(t) ; \forall t \in\{t=1,2, \ldots, T\}\}$ with

$$
G_{(i, j)}(t)=\frac{1}{\Omega} \sum_{\omega=\omega_{a}}^{\omega_{b}} P L V_{(i, j)}(t, \omega),
$$

where $G_{(i, j)}(t) \in[0,1]$ represents the connectivity strength between the nodes $i$ and $j$ within the frequency band of interest, $\left[\omega_{a}, \omega_{b}\right]$, and $\Omega$ is the number of frequency bins in that band.

The time-varying connectivity matrices in this study are constructed by computing the pairwise PLV between 62 channels in the theta frequency band for all time ( 2 seconds corresponding to 256 time samples) and all subjects, resulting in $\mathcal{G}(t) \in \mathbb{R}^{62 \times 62 \times 91}, t \in\{1,2, \ldots, 256\}$ for the error and correct response [54], [55]. The connectivity matrices across subjects at each time point is averaged, which leads to the average connectivity across time $\mathbf{G}(t) \in \mathbb{R}^{62 \times 62}, t \in\{1,2, \ldots, 256\}$.

3) Implementation: The dynamic connectivity networks across time are assumed to follow a time-varying signal plus background model:

$$
\mathbf{G}(t)=\mathbf{B}+\hat{\mathbf{G}}(t),
$$

where $\mathbf{B} \in \mathbb{R}^{62 \times 62}$ is the background FC pattern common across all time steps, and $\hat{\mathbf{G}}(t) \in \mathbb{R}^{62 \times 62}$ is the innovation component of the connectivity matrix at time $t$. Hierarchical DCS is used to find the background FC pattern, $\mathbf{B}$, and 


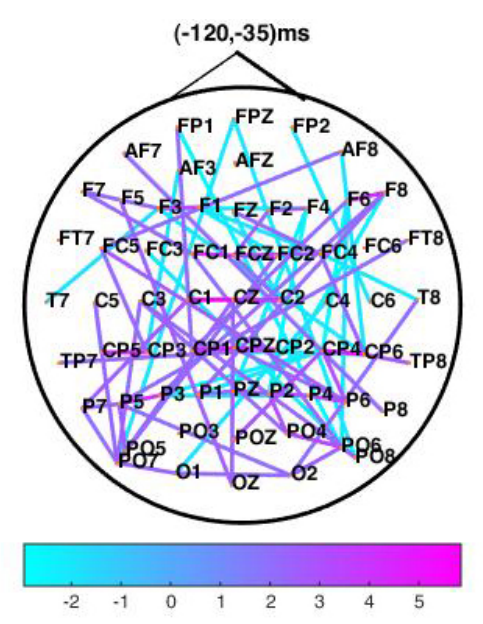

(a)

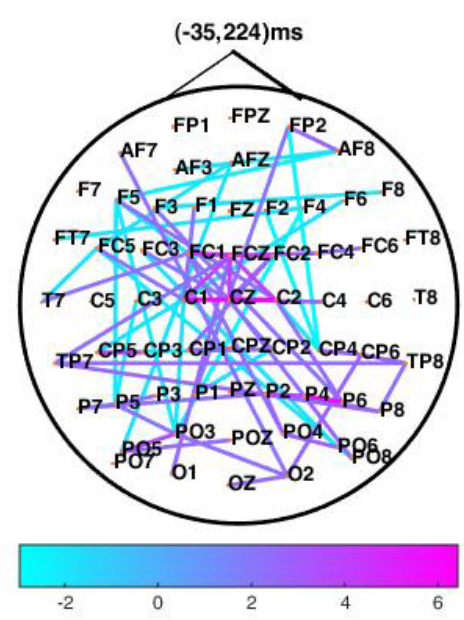

(b)

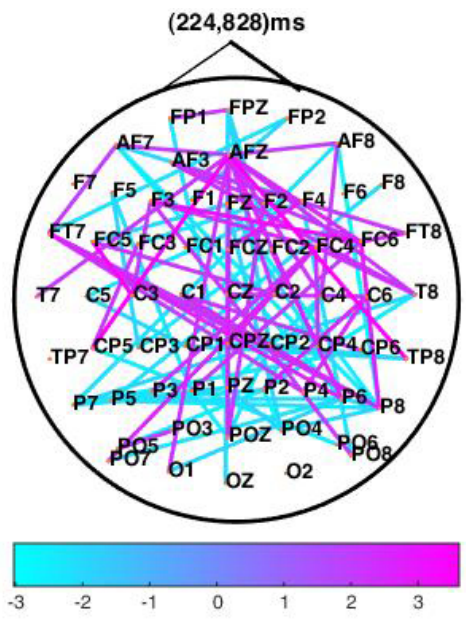

(c)

Fig. 10. The significant edges for the time intervals: (a) $(-120,-35) \mathrm{ms}$, (b) $(-35,224) \mathrm{ms}$, (c) $(224,828) \mathrm{ms,} \mathrm{obtained} \mathrm{through} \mathrm{z-test} \mathrm{with}$ $p=0.95$.

the innovation connectivity matrices, $\hat{\mathbf{G}}(t), t \in\{1,2, \ldots, 256\}$. First, the upper triangular part of the connectivity matrices are vectorized, as:

$$
\mathbf{g}(t)=\mathbf{b}+\hat{\mathbf{g}}(t)
$$

where $\mathbf{g}(t), \mathbf{b}, \hat{\mathbf{g}}(t) \in \mathbb{R}^{1953 \times 1}$. The set of 256 signals, $\mathbf{g}(t), t \in\{1,2, \ldots, 256\}$, is partitioned to $\Gamma=10$ subsets, $\Lambda_{1}=\{\mathbf{g}(1), \ldots, \mathbf{g}(25)\}, \Lambda_{1}=\{\mathbf{g}(26), \ldots, \mathbf{g}(50)\}, \ldots$, and $\Lambda_{10}=\{\mathbf{g}(226), \ldots, \mathbf{g}(256)\}$. Discrete Cosine Transform (DCT) basis is used for the common component, $\phi_{c} \in \mathbb{R}^{1953 \times 1953}$, while Haar wavelet basis is selected for the innovation component, $\phi_{j} \in \mathbb{R}^{1953 \times 1953}$. The MATLAB package "llbenchmark" is used to solve the optimization problem in Eq. 10, where $\mathbf{z}_{c}=\mathbf{b}, \mathbf{x}_{j}=\mathbf{g}(t)$, and $\mathbf{z}_{j}=\hat{\mathbf{g}}(t)$. Once the background FC pattern is determined, Eq. 11 is used to obtain the innovation components, $\hat{\mathbf{g}}(t)$.

Change Point Detection: The innovation components, $\hat{\mathbf{g}}(t)$, are used to detect the change points. To achieve this goal, the support of $\hat{\mathbf{g}}(t)$, i.e. the indices of the non-zero coefficients with respect to the basis of representation, is tracked over time. The FCNs are assumed to be quasi-stationary within a short time interval [56], [57]. Therefore, the support of the innovation components' coefficients should not change much within each time interval. Any change in the support of the coefficients between consecutive time samples is considered as a change point. Let the vector $\tau(t) \in \mathbb{R}^{1953 \times 1}$ be a binary vector such that the $i$ th element $\tau_{i}(t)=1$ if $\hat{\theta}_{j, i}(t)\left(\hat{\theta}_{j, i}(t)\right.$ is the $i$ th element of the vector $\hat{\theta}_{j}$ in Eq. 11 is nonzero, and $\tau_{i}(t)=0$ otherwise. In order to detect the change points, the similarity between the supports of the coefficients of the consecutive time samples is quantified using the Simple Matching Coefficient (SMC) [58]. SMC is a similarity measure between binary vectors, which takes into account both zero and one attributes. Fig. 9 shows the SMC similarity between consecutive $\tau(t) \mathrm{s}$.

From the plot, we can identify several distinct time intervals: $(-1000,-921) \mathrm{ms},(-921,-120) \mathrm{ms},(-120,-35) \mathrm{ms}$, $(-35,224) \mathrm{ms},(224,828) \mathrm{ms}$, and $(828,1000) \mathrm{ms}$. The time interval $(-35,224) \mathrm{ms}$ includes the ERN response. 
During error processing (ERN time interval), the brain is in the process of forming transient frontal networks, which causes the decrease in the similarity of the support of the coefficients of the innovation components of FCNs as the network is changing.

Network Summarization: Once the time intervals are determined, the FC topographic map of each time interval needs to be obtained. FC topographic map consists of the significant functional connections consistent across the detected time interval, $\left(t_{0}, t_{1}\right)$. In order to get the FC topographic map, we apply H-DCS to $\hat{\mathbf{g}}(t), t \in\left(t_{0}, t_{1}\right)$. Thus, the connectivity vectors are written as:

$$
\hat{\mathbf{g}}(t)=\pi+\check{\mathbf{g}}(t), \quad t \in\left(t_{0}, t_{1}\right),
$$

where $\pi$ is the common part of the time interval $\left(t_{0}, t_{1}\right)$ we are interested in, and $\check{\mathbf{g}}(t)$ represents the innovation activity at time $t$.

The signal model assumed for the connectivity matrices within the time interval in Eq. 33 is a common + innovation component joint sparsity model (Eq. 3), where $\mathbf{z}_{c}=\pi, \mathbf{x}_{j}=\hat{\mathbf{g}}(t)$, and $\mathbf{z}_{j}=\check{\mathbf{g}}(t)$. The resulting optimization problem (Eq. 10) for $\hat{\mathbf{g}}(t), t \in\left(t_{0}, t_{1}\right)$ is solved, yielding the common pattern of FCs across time within the time interval, $\pi$. Since the background FC pattern is eliminated in the first step (see Eq. 32), $\pi$ is the common FC pattern specific to the time interval. Since the distribution of the common FC patterns is Gaussian, z-test determines the most significant edges. Fig. 10 shows the most significant FCs for three time intervals, $(-120,-35) \mathrm{ms},(-35,224) \mathrm{ms}$, and $(224,828) \mathrm{ms}$, determined through z-test with $p=0.95$. Fig. 10 (b) corresponds to ERN time interval showing the significant connections between medial-prefrontal and medial-central regions engaging electrodes $\mathrm{FC} 1, \mathrm{FCz}, \mathrm{FC} 2, \mathrm{C} 1, \mathrm{Cz}$, and $\mathrm{C} 2$. This pattern is consistent with widely observed ERN amplitude measures [49], [59], [60]. There are also some connections between occipital-parietal and frontal regions. Fig. 10,a) corresponds to the pre-ERN time interval. In this state, the brain is preparing to respond to the external stimulus with connections distributed across the scalp. There are also some connections among FC1, FCz, and FC2 in addition to the connections among $\mathrm{C} 1, \mathrm{Cz}$, and $\mathrm{C} 2$. Fig. 10 (c) corresponds to the error-related positivity (Pe) and P300, showing strong connections between frontal and parietal regions. Broadly, the network states identified before, during, and after the ERN are consistent with hypothesized ERN-related network dynamics, focused on the formation of transient frontal networks during error processing.

\section{Conclusions}

Joint sparse recovery approximates a set of input signals simultaneously through linear combinations of dictionary elements. The joint sparse recovery algorithm uses the minimum number of bases elements while keeping the error between the approximation and the input signals small. In this paper, we proposed a hierarchical DCS algorithm to jointly recover a set of signals following a sparse common plus innovation model. The hierarchical DCS outperforms the conventional DCS method in terms of the computational time and the memory requirement, significantly. This approach is particularly useful in applications where the number of signals following a joint sparsity model is high. In this paper, we considered two such applications: video background extraction and dynamic functional connectivity brain network tracking and summarization. In both applications, the number of signals, i.e. the number of frames in the first application and the number of time points in the second one, are relatively high and the signals are 
assumed to have a common sparse component across time. H-DCS applied to video data successfully recovered the foreground objects. Similarly, it was shown that this approach provided a way of segmenting the dynamic functional connectivity networks and summarizing the common sparse background activity within each time interval. Future work will consider multi-stage implementations of H-DCS to further reduce the computational time.

\section{REFERENCES}

[1] S. Pazos, M. Hurtado, and C. Muravchik, "On sparse methods for array signal processing in the presence of interference," Antennas and Wireless Propagation Letters, IEEE, vol. 14, pp. 1165-1168, 2015.

[2] J. A. Tropp, A. C. Gilbert, and M. J. Strauss, “Algorithms for simultaneous sparse approximation. part i: Greedy pursuit," Signal Processing, vol. 86, no. 3, pp. 572-588, 2006.

[3] J. A. Tropp, “Algorithms for simultaneous sparse approximation. part ii: Convex relaxation," Signal Processing, vol. 86, no. 3, pp. 589-602, 2006.

[4] A. Rakotomamonjy, "Surveying and comparing simultaneous sparse approximation (or group-lasso) algorithms," Signal processing, vol. 91, no. 7, pp. 1505-1526, 2011.

[5] Z. Luo, M. Gaspar, J. Liu, and A. Swami, "Distributed signal processing in sensor networks," Signal Processing Magazine, IEEE, vol. 23, no. 4, pp. 14-15, 2006.

[6] C. Phillips, J. Mattout, M. D. Rugg, P. Maquet, and K. J. Friston, "An empirical bayesian solution to the source reconstruction problem in eeg," NeuroImage, vol. 24, no. 4, pp. 997-1011, 2005.

[7] I. F. Gorodnitsky, J. S. George, and B. D. Rao, "Neuromagnetic source imaging with focuss: a recursive weighted minimum norm algorithm," Electroencephalography and clinical Neurophysiology, vol. 95, no. 4, pp. 231-251, 1995.

[8] E. Ollila, "Nonparametric simultaneous sparse recovery: an application to source localization," arXiv preprint arXiv:1502.02441, 2015.

[9] M. Fornasier and H. Rauhut, "Recovery algorithms for vector-valued data with joint sparsity constraints," SIAM Journal on Numerical Analysis, vol. 46, no. 2, pp. 577-613, 2008.

[10] J. D. Blanchard, M. Cermak, D. Hanle, and Y. Jing, "Greedy algorithms for joint sparse recovery," Signal Processing, IEEE Transactions on, vol. 62, no. 7, pp. 1694-1704, 2014.

[11] D. Baron, M. B. Wakin, M.F. Duarte, S. Sarvotham, and R.G. Baraniuk, "Distributed compressed sensing," 2005.

[12] D. Baron, M. F. Duarte, M. B. Wakin, S. Sarvotham, and R.G. Baraniuk, "Distributed compressive sensing," arXiv preprint arXiv:0901.3403, 2009.

[13] L. Kang and C. Lu, "Distributed compressive video sensing," in Acoustics, Speech and Signal Processing, 2009. ICASSP 2009. IEEE International Conference on. IEEE, 2009, pp. 1169-1172.

[14] T.T. Do, Y. Chen, D.T. Nguyen, N. Nguyen, L. Gan, and T.D. Tran, "Distributed compressed video sensing," in Image Processing (ICIP), 2009 16th IEEE International Conference on. IEEE, 2009, pp. 1393-1396.

[15] Y. Baig, E. Lai, and A. Punchihewa, "Distributed video coding based on compressed sensing," in Multimedia and Expo Workshops (ICMEW), 2012 IEEE International Conference on. IEEE, 2012, pp. 325-330.

[16] S. Patterson, Y.C. Eldar, and I. Keidar, "Distributed compressed sensing for static and time-varying networks," Signal Processing, IEEE Transactions on, vol. 62, no. 19, pp. 4931-4946, 2014.

[17] K. Lee, Y. Bresler, and M. Junge, "Subspace methods for joint sparse recovery," IEEE Transactions on Information Theory, vol. 58, no. 6, pp. 3613-3641, 2012.

[18] M. Mishali and Y.C. Eldar, "Reduce and boost: Recovering arbitrary sets of jointly sparse vectors," IEEE Transactions on Signal Processing, vol. 56, no. 10, pp. 4692-4702, 2008.

[19] X. Yuan, X. Liu, and S. Yan, "Visual classification with multitask joint sparse representation," IEEE Transactions on Image Processing, vol. 21, no. 10, pp. 4349-4360, 2012. 
[20] M. Hyder and K. Mahata, "A robust algorithm for joint-sparse recovery," IEEE Signal Processing Letters, vol. 16, no. 12, pp. 1091-1094, 2009.

[21] X. Zhang, T. Huang, Y. Tian, and W. Gao, "Background-modeling-based adaptive prediction for surveillance video coding," Image Processing, IEEE Transactions on, vol. 23, no. 2, pp. 769-784, 2014.

[22] A. Manzanera and J.C. Richefeu, "A new motion detection algorithm based on $\sigma-\delta$ background estimation," Pattern Recognition Letters, vol. 28 , no. 3 , pp. 320-328, 2007.

[23] D. Comaniciu, V. Ramesh, and P. Meer, "Kernel-based object tracking," Pattern Analysis and Machine Intelligence, IEEE Transactions on, vol. 25, no. 5, pp. 564-577, 2003.

[24] A. Yilmaz, O. Javed, and M. Shah, "Object tracking: A survey," Acm computing surveys (CSUR), vol. 38, no. 4, pp. 13 , 2006.

[25] F. Babiloni, F. Cincotti, C. Babiloni, F. Carducci, D. Mattia, L. Astolfi, A. Basilisco, P.M. Rossini, L. Ding, Y. Ni, J. Chengg, K. Christineg, J. Sweeneyg, and B. He, "Estimation of the cortical functional connectivity with the multimodal integration of high-resolution eeg and fmri data by directed transfer function," Neuroimage, vol. 24, no. 1, pp. 118-131, 2005.

[26] F. Varela, J.P. Lachaux, E. Rodriguez, and J. Martinerie, "The brainweb: Phase synchronization and large-scale integration," Nature Reviews Neuroscience, vol. 2, no. 4, pp. 229-239, 2001.

[27] J. Lachaux, E. Rodriguez, J. Martinerie, and F.J. Varela, "Measuring phase synchrony in brain signals," Human brain mapping, vol. 8, no. 4, pp. 194-208, 1999.

[28] E. Bullmore and O. Sporns, "Complex brain networks: graph theoretical analysis of structural and functional systems," Nature Reviews Neuroscience, vol. 10, no. 3, pp. 186-198, 2009.

[29] M. P. van den Heuvel, C.J. Stam, M. Boersma, and H.E.H. Pol, "Small-world and scale-free organization of voxel-based resting-state functional connectivity in the human brain," Neuroimage, vol. 43, no. 3, pp. 528-539, 2008.

[30] C.J. Stam and J.C. Reijneveld, "Graph theoretical analysis of complex networks in the brain," Nonlinear biomedical physics, vol. 1, no. 1, pp. 3, 2007.

[31] R. Prabhakaran, S.E. Blumstein, E.B. Myers, E. Hutchison, and B. Britton, "An event-related fmri investigation of phonological-lexical competition," Neuropsychologia, vol. 44, no. 12, pp. 2209-2221, 2006.

[32] F. Freyer, K. Aquino, P. A. Robinson, P. Ritter, and M. Breakspear, "Bistability and non-gaussian fluctuations in spontaneous cortical activity," The Journal of Neuroscience, vol. 29, no. 26, pp. 8512-8524, 2009.

[33] C. Chang and G.H. Glover, "Time-frequency dynamics of resting-state brain connectivity measured with fmri," Neuroimage, vol. 50, no. 1, pp. 81-98, 2010.

[34] N. Leonardi, W. R. Shirer, M. D. Greicius, and D. Van De Ville, "Disentangling dynamic networks: Separated and joint expressions of functional connectivity patterns in time," Human brain mapping, vol. 35, no. 12, pp. 5984-5995, 2014.

[35] E.A. Allen, E. Damaraju, S.M. Plis, E.B. Erhardt, T. Eichele, and V. D. Calhoun, "Tracking whole-brain connectivity dynamics in the resting state," Cerebral cortex, p. bhs352, 2012.

[36] K. Marcotte, V. Perlbarg, G. Marrelec, H. Benali, and A.I. Ansaldo, "Default-mode network functional connectivity in aphasia: therapyinduced neuroplasticity," Brain and language, vol. 124, no. 1, pp. 45-55, 2013.

[37] M. F. Duarte, S. Sarvotham, D. Baron, M.B. Wakin, and R.G. Baraniuk, "Distributed compressed sensing of jointly sparse signals," in Asilomar Conf. Signals, Sys., Comput, 2005, pp. 1537-1541.

[38] D. Baron, M.F. Duarte, S. Sarvotham, M. B. Wakin, and R.G. Baraniuk, "An information-theoretic approach to distributed compressed sensing," in Proc. 45rd Conference on Communication, Control, and Computing, 2005.

[39] S. Sarvotham M. B. Wakin R. G. Baraniuk D. Baron, M. Duarte, "Distributed compressive sensing," Tech. Rep. TREE0612, 2006, http://dsp.rice.edu/cs/.

[40] D.L. Donoho, Y. Tsaig, I. Drori, and J. Starck, "Sparse solution of underdetermined systems of linear equations by stagewise orthogonal matching pursuit," Information Theory, IEEE Transactions on, vol. 58, no. 2, pp. 1094-1121, 2012. 
[41] E.J. Candes, J.K. Romberg, and T. Tao, "Stable signal recovery from incomplete and inaccurate measurements," Communications on pure and applied mathematics, vol. 59, no. 8, pp. 1207-1223, 2006.

[42] H. Rauhut, K. Schnass, and P. Vandergheynst, "Compressed sensing and redundant dictionaries," Information Theory, IEEE Transactions on, vol. 54, no. 5, pp. 2210-2219, 2008.

[43] D. Needell and R. Vershynin, "Signal recovery from incomplete and inaccurate measurements via regularized orthogonal matching pursuit," IEEE Journal of selected topics in signal processing, vol. 4, no. 2, pp. 310-316, 2010.

[44] O. Barnich and M. Van Droogenbroeck, "Vibe: a powerful random technique to estimate the background in video sequences," in Acoustics, Speech and Signal Processing, 2009. ICASSP 2009. IEEE International Conference on. IEEE, 2009, pp. 945-948.

[45] “The usc-sipi image database: Image sequence," Accessed: 2015-07-31, http://sipi.usc.edu/database/ .

[46] J. R. Hall, E. M. Bernat, and C. J. Patrick, "Externalizing psychopathology and the error-related negativity," Psychological Science, vol. 18, no. 4, pp. 326-333, 2007.

[47] J. Kayser and C.E. Tenke, "Principal components analysis of laplacian waveforms as a generic method for identifying ERP generator patterns: I. evaluation with auditory oddball tasks," Clinical Neurophysiology, vol. 117, no. 2, pp. 348-368, 2006.

[48] J.R. Hall, E.M. Bernat, and C.J. Patrick, "Externalizing psychopathology and the error-related negativity," Psychological Science, vol. 18, no. 4, pp. 326-333, 2007.

[49] J.F. Cavanagh, M.X. Cohen, and J.J.B. Allen, "Prelude to and resolution of an error: EEG phase synchrony reveals cognitive control dynamics during action monitoring," The Journal of Neuroscience, vol. 29, no. 1, pp. 98-105, 2009.

[50] S. Aviyente, E.M. Bernat, W.S. Evans, and S.R. Sponheim, "A phase synchrony measure for quantifying dynamic functional integration in the brain," Human Brain Mapping, vol. 32, no. 1, pp. 80-93, 2011.

[51] M. Steriade, P. Gloor, R.R. Llinas, F.H. Lopes Da Silva, and M. Mesulam, "Basic mechanisms of cerebral rhythmic activities," Electroencephalography and clinical neurophysiology, vol. 76, no. 6, pp. 481-508, 1990.

[52] S. L. Bressler, "Large-scale cortical networks and cognition," Brain Research Reviews, vol. 20, no. 3, pp. 288-304, 1995.

[53] S. Aviyente and A.Y. Mutlu, "A time-frequency-based approach to phase and phase synchrony estimation," IEEE Transactions on Signal Processing, vol. 59, no. 7, pp. 3086-3098, 2011.

[54] A. G. Mahyari and S. Aviyente, "A tucker decomposition based approach for topographic functional connectivity state summarization," in Proceedings of the 3rd IEEE Global Conference on Signal and Information Processing (GlobalSIP).

[55] A. G. Mahyari and S. Aviyente, "Identification of dynamic functional brain network states through tensor decomposition," in Acoustics, Speech and Signal Processing (ICASSP), 2014 IEEE International Conference on. IEEE, 2014, pp. 2099-2103.

[56] R.F. Betzel, M.A. Erickson, M. Abell, B.F. O’Donnell, W.P. Hetrick, and O. Sporns, "Synchronization dynamics and evidence for a repertoire of network states in resting eeg," Frontiers in computational neuroscience, vol. 6, 2012.

[57] M.J. Lowe, E.B. Beall, K.E. Sakaie, K.A. Koenig, L. Stone, R.A. Marrie, and M.D. Phillips, "Resting state sensorimotor functional connectivity in multiple sclerosis inversely correlates with transcallosal motor pathway transverse diffusivity," Human brain mapping, vol. 29 , no. 7 , pp. 818-827, 2008.

[58] J.C. Gower, “A general coefficient of similarity and some of its properties," Biometrics, pp. 857-871, 1971.

[59] M. Bolanos, E.M. Bernat, B. He, and S. Aviyente, "A weighted small world network measure for assessing functional connectivity," Journal of neuroscience methods, vol. 212, no. 1, pp. 133-142, 2013.

[60] A. Y. Mutlu, E. Bernat, and S. Aviyente, "A signal-processing-based approach to time-varying graph analysis for dynamic brain network identification," Computational and Mathematical Methods in Medicine, vol. 2012, 2012. 